\title{
Searching for low mass dark matter via phonon creation in superfluid ${ }^{4} \mathrm{He}$
}

\author{
Gordon Baym® ${ }^{1,2}$ D. H. Beck $\odot,{ }^{1}$ Jeffrey P. Filippini $\odot,{ }^{1}$ C. J. Pethick $\odot,{ }^{1,2,3}$ and Jessie Shelton ${ }^{1}$ \\ ${ }^{1}$ Illinois Center for Advanced Studies of the Universe and Department of Physics, \\ University of Illinois at Urbana-Champaign, Urbana, Illinois 61801, USA \\ ${ }^{2}$ The Niels Bohr International Academy, The Niels Bohr Institute, University of Copenhagen, \\ Blegdamsvej 17, DK-2100 Copenhagen Ø, Denmark \\ ${ }^{3}$ NORDITA, KTH Royal Institute of Technology and Stockholm University, \\ Roslagstullsbacken 23, SE-10691 Stockholm, Sweden
}

(Received 18 May 2020; accepted 17 July 2020; published 11 August 2020)

\begin{abstract}
We consider the scattering of dark matter particles from superfluid liquid ${ }^{4} \mathrm{He}$, which has been proposed as a target for their direct detection. Focusing on dark matter masses below $\sim 1 \mathrm{MeV}$, we demonstrate from sum-rule arguments the importance of the production of single phonons with energies $\omega \lesssim 1 \mathrm{meV}$. We show further that the anomalous dispersion of phonons in liquid ${ }^{4} \mathrm{He}$ at low pressures [i.e., $d^{2} \omega(q) / d q^{2}>0$, where $q$ and $\omega(q)$ are the phonon momentum and energy] has the important consequence that a single phonon will decay over a relatively short distance into a shower of lower-energy phonons centered on the direction of the original phonon. Thus, the experimental challenge in this regime is to detect a shower of low-energy phonons, not just a single phonon. Additional information from the distribution of phonons in such a shower could enhance the determination of the dark matter mass.
\end{abstract}

DOI: 10.1103/PhysRevD.102.035014

\section{INTRODUCTION}

Although the existence of dark matter has been conclusively established by multiple independent lines of gravitational evidence [1], its nature remains one of the outstanding mysteries in physics. Weakly Interacting Massive Particle (WIMP) models of dark matter, which rely on Standard Model interactions to produce the dark matter relic abundance, have thus been an important experimental target for decades [2,3]. A broad range of experiments has been deployed to directly detect the elastic scattering of $\sim 10 \mathrm{GeV}$-mass WIMPs from heavy nuclei (see, e.g., Ref. [4] and references therein).

With WIMP dark matter now under increasing observational strain, broadening the scope of terrestrial searches for dark matter has become ever more compelling $[5,6]$. If dark matter interacts with the matter of the Standard Model via a new, dark force, the mass range of interest for direct detection experiments becomes much broader, and in particular extends down to the observational warm dark matter limit of order a few $\mathrm{keV}$ [7]. The direct detection of sub-MeV dark matter poses substantial challenges, due to the poor kinematic match with atomic nuclei and the very

Published by the American Physical Society under the terms of the Creative Commons Attribution 4.0 International license. Further distribution of this work must maintain attribution to the author(s) and the published article's title, journal citation, and DOI. Funded by SCOAP ${ }^{3}$. low available kinetic energy, $<1 \mathrm{eV}$ for sub-MeV dark matter moving at typical galactic velocities $\left(v / c \sim 10^{-3}\right)$. Many interesting new or proposed experiments aim at dark matter masses $m_{\chi}$ in the range from $\mathrm{MeV}$ to a few $\mathrm{GeV}$, either using electronic scattering [8] in a variety of systems such as semiconductors [9-13], liquid noble gases [14-16], and other materials $[17,18]$; or using new channels to observe nuclear scattering [19-22]. Far fewer experiments have been proposed to detect dark matter in the challenging sub-MeV regime. Such schemes generally involve systems with very low-energy gapped excitations-e.g., quasiparticles in superconductors [23], electrons in Dirac materials [24], and optical phonons in polar crystals [25]. More recently, Ref. [26] has called attention to the advantages of using materials with high sound speeds.

Superfluid ${ }^{4} \mathrm{He}$ is a particularly promising target for the detection of light dark matter particles. Atomic helium recoils from $\mathrm{GeV}$-mass particles can be detected via the resulting electronic excitations, visible as scintillation and ionization [27]. Lighter particles can excite phonons and rotons, the meV-scale collective excitations of the superfluid, as discussed in detail by Schutz and Zurek [28] and by Knapen, Lin, and Zurek [29]. Such excitations may further evaporate individual ${ }^{4} \mathrm{He}$ atoms from the superfluid surface, forming the basis of a detection scheme proposed by Maris et al. [30,31] and recently explored by Hertel et al. [32].

In this paper, we explore in detail the physics of excitations produced by the scattering of dark matter 
particles in superfluid helium, focusing on the challenging mass range $m_{\chi} \lesssim 1 \mathrm{MeV}$. Such dark matter particles are not energetic enough to excite helium atoms electronically. We formulate the interaction between the dark matter particle $\chi$ and ${ }^{4} \mathrm{He}$ atoms in terms of a low-energy $s$-wave pseudopotential-essentially a contact interaction. Predicting the interaction rate then reduces to understanding the density fluctuations in the helium, which at low energy are single phonons and rotons, as well as multiple phonon and roton excitations. Through the use of sum rules and explicit calculations, we constrain the production and damping of these excitations across a wide kinematic range. Our focus throughout is on deriving the response of the superfluid, rather than on proposing a detector design or dark matter model.

The present analysis extends that of Zurek et al. [28,29] in two significant ways. First, these authors limited themselves to processes that could generate phonons with energies above $1 \mathrm{meV}$, an assumed detection limit. Since the maximum phonon-roton energy (the maxon) is $\sim 1.1 \mathrm{meV}$, this cut effectively excludes single-phonon processes and requires multiphonon excitations. They draw upon theoretical calculations of high-frequency density fluctuations [33] in estimating detection rates. Here we consider the generation and propagation of excitations over a broader range of excitation energies, leaving aside for the moment issues of detectability. As we discuss more fully below, the $f$-sum rule for phonon fluctuations implies that as the phonon momentum decreases, single-phonon processes become more and more dominant, exhausting some $90 \%$ of the allowed weight even at the highest momentum transfer.

A second feature we take into account here is the important role played by anomalous dispersion: the slight deviation from linearity of the low-energy phonon spectrum. Anomalous dispersion allows single low-momentum phonons to decay into two (or more) phonons nearly collinear with the initial phonon-the Beliaev process $[34,35]$. As we show in detail, this process leads to the rapid formation of phonon cones, analogues of cosmic ray air showers in the atmosphere, after the creation of a single phonon. ${ }^{1}$ Although the detection of such soft phonon cascades is extremely challenging, their shape and extent at the helium surface encodes information on the location of the initial interaction and momentum direction beyond that available from a calorimetric measurement of the initial phonon.

This paper is organized as follows: In the next section we introduce the cast of characters: the dark matter halo in the

\footnotetext{
${ }^{1}$ Acanfora et al. present an effective field theory approach to the problem of detecting sub-GeV dark matter in superfluid ${ }^{4} \mathrm{He}$ [36], in which the phonon dispersion relation is purely linear. While parts of that discussion parallel the treatment here, their approach does not account for the physics of anomalous dispersion. See also Ref. [37,38].
}

neighborhood of the Earth, and the excitations of superfluid ${ }^{4} \mathrm{He}$. We then, in Sec. III, review the kinematics of the interaction between dark matter particles and the helium, and we model their interaction in terms of a low-energy pseudopotential. In the following Sec. IV, we show that the $f$-sum rule bounds the rate of multiexcitation emission compared with the single-phonon rate to at most $10 \%$ at $q \lesssim 0.35 \AA^{-1}$, determine the rate of single-phonon emission, and describe phonon splitting and damping in the anomalous dispersion regime. We discuss phonon damping in Sec. V, two-phonon production in Sec. VI, and turn in the following Sec. VII to describing the phonon cascade produced by an initial single phonon of low $q$, and we outline how the detection of such a cascade would proceed. Appendix A is devoted to a technical discussion of the relation of the helium structure function, $S(q, \omega)$, and the helium density-density correlation function, Appendix B discusses $S(q, \omega)$ at nonzero temperature, and Appendix C discusses the $q$ dependence of the rate of direct production of a pair of phonons.

\section{PHYSICAL SETTING}

The problem of dark matter scattering in superfluid helium lies at the intersection of quite disparate threads of physics, spanning decades of literature across different research communities. In this section, we briefly review the groundwork we need on two key topics: the flux and velocity distribution of dark matter incident upon the Earth, and the basic phenomenology of collective excitations in superfluid helium.

\section{A. Dark matter halo}

Estimating the rates and spectra of interactions between dark matter and Earth-bound systems requires a model of the density and velocity distribution of dark matter particles in our local neighborhood. Interaction rates are directly proportional to the local dark matter density, a relatively uncertain quantity (see, e.g., Refs. [39-41]); we adopt here $\rho \simeq 0.4 \mathrm{GeV} / \mathrm{cm}^{3}$. The velocity distribution of dark matter, $f(v) d v$ (normalized to unity), in the solar neighborhood is typically modeled as Maxwell-Boltzmann, cut off at some Galactic escape velocity, $v_{\text {esc }}$; here we assume a characteristic velocity $v_{0}=230 \mathrm{~km} / \mathrm{s}[42,43]$ in the Galactic frame, with $v_{\mathrm{esc}}=550 \mathrm{~km} / \mathrm{s}$ [44]. This velocity distribution is further boosted into the rest frame of the Sun $\left(v_{E}=244 \mathrm{~km} / \mathrm{s}\right)$, with further modulations from the Earth's motion around the Sun neglected here [45,46]. Dark matter particles are thus incident upon terrestrial detectors at typical velocities of magnitude $v \sim 300 \mathrm{~km} / \mathrm{s}$, but have significantly higher and lower speeds.

This simple form for the velocity distribution, the "standard halo model," is a useful first approximation to the local dynamics of dark matter; its common use enables straightforward comparison between different experimental 
probes of dark matter. However, the actual phase space distribution of dark matter at the Earth is poorly determined experimentally, and we should not expect the standard halo model to yield a precise description of the local dark matter distribution. First, numerical simulations of Milky Waylike galaxies typically predict velocity distributions broader than Maxwellian, with more support at large speeds [47]. Second, the Milky Way is not in a steady state: smaller systems are continuously accreting onto the Milky Way, giving rise to substructure in the phase space distribution of dark matter, which can produce localized enhancements of dark matter at relatively high velocities $[48,49]$.

The total elastic scattering rate evaluated at the mean velocity and the rate integrated over the full velocity distribution differ only by factors of order 1 . However, the total rates above some specified threshold can be much more sensitive to the form of the velocity distribution, depending on where the threshold falls relative to the mean of the distribution. In particular, velocity averaging is critical for determining the lowest dark matter masses that a given experiment is sensitive to, and will be a necessary component of any tradeoff made between, e.g., measuring lower-energy single-phonon signals versus exposing a higher-threshold detector for longer times. For convenience, we will typically quote parameters at a single representative velocity, but integrate over the standard halo model when giving total rates in Secs. IV and VI.

\section{B. Helium excitations}

The de Broglie wavelength, $\lambda_{d B}=2 \pi \hbar / m_{\chi} v$, of a dark matter particle of mass $m_{\chi}$ between $10 \mathrm{keV}$ and $1 \mathrm{MeV}$, of order 2000 to $20 \AA$, is much larger than the average spacing, $4.5 \AA$, between $\mathrm{He}$ atoms in the superfluid. Therefore, the $\chi$ 's are scattered by the helium via the creation of collective modes of the superfluid-the phonons and rotons. The familiar phonon-roton dispersion curve is shown in Fig. 1 [50]. The detection of ${ }^{4} \mathrm{He}$ excitations created by a $\chi$ and the measurement of their energies and directions with respect to $\vec{v}$ are adequate to learn the mass of the initial dark matter particle, as well as the $\chi^{-}{ }^{4} \mathrm{He}$ cross section. In this paper, we focus primarily on phonons.

The phonon dispersion relation, energy vs momentum, is approximately $\omega \simeq s q$, where $s=2.38 \times 10^{4} \mathrm{~cm} / \mathrm{s}=$ $1.56 \mathrm{meV} \AA$ (at saturated vapor pressure, SVP) is the speed of sound in the superfluid. We take $\hbar=1$ throughout. Dark matter particles thus move at supersonic velocity, $v \sim 10^{3} \mathrm{~s}$. Importantly, between saturated vapor pressure (SVP) and $\sim 18$ bar [51-53], the phonon dispersion relation curves slightly upward initially:

$$
\omega(q) \simeq s q\left(1+\zeta_{A} q^{2}+\cdots\right),
$$

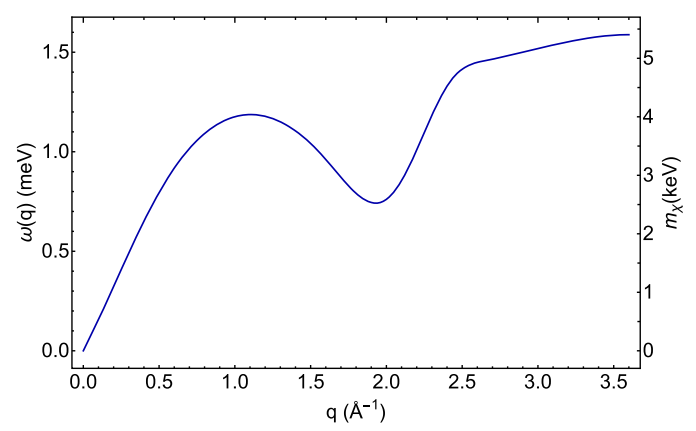

FIG. 1. The dispersion curve of superfluid helium [50]. The excitations in the range below about $q=1 \AA^{-1}$ are phonons, and in the vicinity of $q=1.9 \AA^{-1}$ are rotons. Although not visible on this scale, the second derivative of the dispersion curve is positive (anomalous dispersion) below momenta $q_{\text {infl }}$; at SVP $q_{\text {infl }} \approx 0.216 \AA^{-1}$. At pressure above $\sim 18$ bar, the anomalous dispersion vanishes. The scale on the right indicates the dark matter mass, $m_{\chi}$, with kinetic energy corresponding to the scale on the left, for dark matter particles moving at (minus) the velocity, $v$, of the Solar System through the Galaxy.

an effect known as anomalous dispersion. At SVP, the upward curvature stops at phonon momentum $q_{\text {infl }} \approx 0.216 \AA^{-1}$, where the dispersion relation has an inflection point.

A more detailed parametrization of the phonon dispersion relation than Eq. (1), valid up to $q \sim 0.9 \AA^{-1}$, is given by Maris [35],

$$
\omega(q) \simeq s q\left(1+\zeta_{A} q^{2}\left(\frac{1-q^{2} / q_{a}^{2}}{1+q^{2} / q_{b}^{2}}\right)\right)
$$

with the parameters $\zeta_{A}=1.11 \AA^{2}, q_{a}=0.542 \AA^{-1}$, and $q_{b}=0.332 \AA^{-1}$ at SVP; see also Refs. [56,57]. The parametrization (2) includes the negative curvature of the dispersion relation at higher $q$, but it does not accurately describe the peak in the dispersion relation, as shown in Fig. 2. At higher phonon momentum, we will use the simple parametrization [58]

$$
\omega(q) \simeq s q\left(1-\zeta_{N} q^{2}+\cdots\right)
$$

with $\zeta_{N} \simeq 0.27 \AA^{2}$.

\footnotetext{
${ }^{2}$ Expression (2) implicitly assumes that $\omega(q)^{2}$ is analytic in $q^{2}$, and consequently that a power series expansion of $\omega(q)$ has only odd powers of $q$. However, as pointed out by Kemoklidze and Pitaevskii [54], the $r^{-6}$ falloff of the van der Waals interaction between helium atoms implies that $\omega(q)$ has even powers of $q$, beginning with $q^{4}$. More recently, approximations to the excitation spectrum that also contain even powers of $q$ have been made (see, e.g., Ref. [55]). The addition of such terms to the dispersion relation is not expected to alter the basic picture we develop, so to simplify the discussion we do not take them into account explicitly.
} 

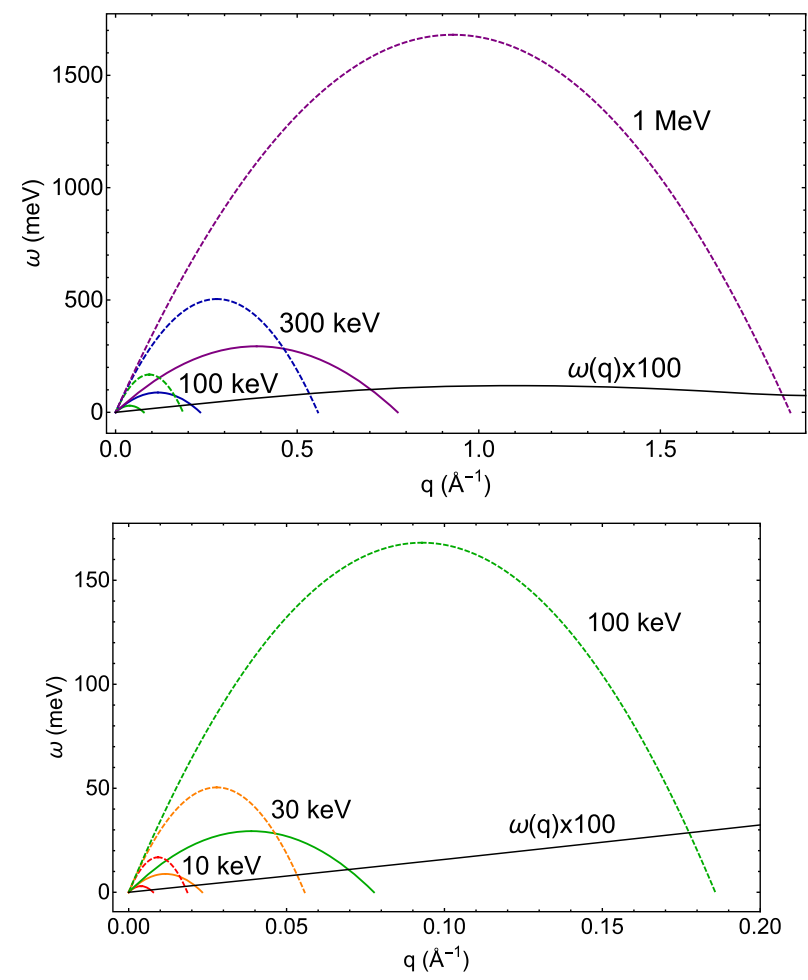

FIG. 2. The region of energy and momentum deposition allowed by Eq. (4) for a dark matter particle of mass $m_{\chi}$, lies within the inverted parabolas, labeled by $m_{\chi}=1 \mathrm{MeV}, 300 \mathrm{keV}$, $100 \mathrm{keV}$ (upper panel), and $m_{\chi}=100 \mathrm{keV}, 30 \mathrm{keV}$, and $10 \mathrm{keV}$ (lower panel). The solid lines assume velocity $v=230 \mathrm{~km} / \mathrm{s}$, and the dashed curves assume $v=550 \mathrm{~km} / \mathrm{s}$. For the creation of a single phonon by the dark matter particle, the energy-momentum transfer lies on the single-phonon dispersion curve (Fig. 1), shown here multiplied in energy by 100 . Only the $m_{\chi}=1 \mathrm{MeV}$ curve, for $v=550 \mathrm{~km} / \mathrm{s}$, extends beyond the maximum in the phonon dispersion curve.

Owing to anomalous dispersion, single-phonon final states are stable against decay only for $q$ larger than a critical value, $q_{c}$, which depends on the details of the dispersion relation. For the Maris dispersion relation [Eq. (2)], $q_{c}=0.4215 \AA^{-1}$, with $\omega\left(q_{c}\right)=7.90 \mathrm{~K}=0.68 \mathrm{meV}$. (A phonon of momentum $q_{c}$ can decay into two collinear equal-momenta phonons, $\omega\left(q_{c}\right)=2 \omega\left(q_{c} / 2\right)$; see details in Sec. V.) Phonons produced with $q<q_{c}$ generate a cascade of lower-momentum phonons.

\section{DARK MATTER SCATTERING ON SUPERFLUID HELIUM}

We first lay out the region of possible energy and momentum transfer, $\omega$ and $\vec{q}$, from a dark matter particle, $\chi$, to the helium. The allowed energy transfer vs momentum transfer is shown in Fig. 2 for representative $m_{\chi}$. For initial momentum $\vec{k}=m_{\chi} \vec{v}$ of the $\chi$, the final momentum is $\vec{k}^{\prime}=\vec{k}-\vec{q}$, and the energy transfer is

$$
\omega=\vec{v} \cdot \vec{q}-\frac{q^{2}}{2 m_{\chi}} .
$$

For $v \simeq 230 \mathrm{~km} / \mathrm{s}$, the incident momentum is $k \simeq$ $0.39 m_{\mathrm{MeV}} \AA^{-1}$, where $m_{\mathrm{MeV}}$ is the mass of the $\chi$ measured in $\mathrm{MeV}$. The maximum energy transfer for given $q$ occurs when $\vec{q}$ is parallel to the incident $\vec{k}$; then $\omega_{\max }(q)=$ $\left(k q-q^{2} / 2\right) / m_{\chi}$, an inverted parabola ranging from 0 to $2 k$ along the $q$ axis, with a maximum at $k=q$ and height $\omega_{\max }(k)=k^{2} / 2 m_{\chi}$, which is the maximum energy transfer from the dark matter particle. The momentum transfer ranges from 0 to $q_{\max }=2 k$, the latter for backscattering and no energy transfer to the medium.

Since the energy-momentum transfers of the dark matter particle to the liquid ${ }^{4} \mathrm{He}$ are so much smaller than the scales associated with the expected microscopic interactions of dark matter with the ${ }^{4} \mathrm{He}$ nuclei or with the electrons, the scattering is primarily $s$-wave, and the interaction of a dark matter particle with a ${ }^{4} \mathrm{He}$ atom can be described by a low energy pseudopotential, ${ }^{3}$

$$
V_{\chi_{4}}=\frac{2 \pi a}{m_{r}} \delta\left(\vec{r}_{\chi}-\vec{r}_{4}\right)
$$

where $a$ is the scattering length and $m_{r}$ is the dark matter $-{ }^{4} \mathrm{He}$ reduced mass; for $m_{\chi} \ll m_{4}$, the ${ }^{4} \mathrm{He}$ mass, $m_{r} \simeq m_{\chi}$. The total cross section for scattering of a dark matter particle on an isolated ${ }^{4} \mathrm{He}$ atom is $\sigma_{\chi_{4}}=4 \pi a^{2}$. The pseudopotential modifies the energy of a dark matter particle in liquid ${ }^{4} \mathrm{He}$ by $2 \pi a n_{4} / m_{r}$, where $n_{4}$ is the ${ }^{4} \mathrm{He}$ equilibrium number density $=2.379 \times 10^{22} \mathrm{~cm}^{-3}$. More complicated dependence of the dark matter particle energy on $n_{4}$ and the ${ }^{4} \mathrm{He}$ velocity fluctuations can be ignored, since the interaction of the $\chi$ with the ${ }^{4} \mathrm{He}$ is weak.

The differential rate at which dark matter particles of density $n_{\chi}$ deposit energy $\omega$ and momentum $q$ in the ${ }^{4} \mathrm{He}$ is given in terms of the dynamical structure function of the ${ }^{4} \mathrm{He}$ by

\footnotetext{
${ }^{3}$ If the interaction of the $\chi$ with baryonic matter is mediated by a (dark) meson of mass $\mu$, then in lowest order the interaction is a Yukawa-like potential, $V_{\mu}(r)=a \mu^{2} e^{-\mu r} / r$, with a scattering amplitude of the form $a /\left(1+(\mu q)^{2}\right)$, where $q$ is the momentum transfer from the dark matter to the helium. The assumption of a simple pseudopotential is valid for $q \ll \mu$, in which case the scattering amplitude is simply $a$. On the other hand, if $\mu$ is smaller than the range of observable phonon momenta, $\mu \ll q$, then the scattering amplitude would behave as $a \mu^{2} / q^{2}$, increasing strongly with decreasing $q$. If one places an upper bound on the scattering amplitude by measurements involving momentum transfers $q_{0}$ larger than typical phonon momenta, then lower-momentum processes can have a scattering amplitude larger by a factor $\sim\left(1+q_{0}^{2} / \mu^{2}\right) /\left(1+q^{2} / \mu^{2}\right)$ than the bound. For $q, q_{0} \ll m_{0}$, the growth in amplitude, $\sim q_{0}^{2} / q^{2}$, can be significant.
} 


$$
d \Gamma=n_{\chi}\left(\frac{2 \pi a}{m_{\chi}}\right)^{2} 2 \pi n_{4} S(q, \omega) \frac{d^{3} q}{(2 \pi)^{3}},
$$

where $\omega$ is given by Eq. (4), and the dynamical structure function is

$$
\begin{aligned}
2 \pi n_{4} S(q, \omega) & =\int d^{3} r d t e^{-i \vec{q} \cdot\left(\vec{r}-\vec{r}^{\prime}\right)+i \omega\left(t-t^{\prime}\right)}\left\langle\rho(\vec{r}, t) \rho\left(\vec{r}^{\prime}, t^{\prime}\right)\right\rangle \\
& =\sum_{f}\left|\left\langle f\left|\rho_{-\vec{q}}\right| i\right\rangle\right|^{2} 2 \pi \delta\left(\omega-E_{f}+E_{i}\right),
\end{aligned}
$$

with $\rho$ the ${ }^{4} \mathrm{He}$ number density operator and $\rho_{q}=$ $\int d^{3} r e^{-i \vec{q} \cdot \vec{r}} \rho(\vec{r}, t)$. The states $i$ and $f$ are those of ${ }^{4} \mathrm{He}$ in equilibrium, in the absence of dark matter, and a thermal average over states $i$ is assumed at nonzero temperature. We assume $\omega>0$ always.

The Fourier transform of the ${ }^{4} \mathrm{He}$ number density operator, $\rho_{-\vec{q}}$, acting on a state of the liquid, can create one or more elementary excitations of the fluid of total momentum $\vec{q}$, or annihilate excitations of total momentum $-\vec{q}$. Thus, a dark matter particle interacting with the ${ }^{4} \mathrm{He}$ can create one or more excitations of the superfluid. The creation of a single phonon is illustrated in Fig. 3(a). This phonon can more generally transform into two or more phonons via the multiphonon interactions in ${ }^{4} \mathrm{He}$ [Fig. 3(b)]. In addition, a $\chi$ can directly create a pair of phonons, as shown in Fig. 3(c). We note that successive creations of phonons by a dark matter particle, as in Fig. 3(d), are higher order in the dark matter-helium scattering length and can be ignored.

The rate in Eq. (6) is independent of the azimuthal angle of $\vec{q}$ about $\vec{v}$. Since the energy $\omega$ is linear in $\cos \theta_{q}$, where $\theta_{q}$ is the angle between $\vec{q}$ and $\vec{v}$, we may replace the differential $d \cos \theta_{q}$ with $d \omega / q v$ and rewrite the rate as

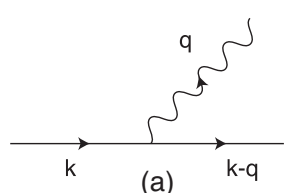

(a)

(c)

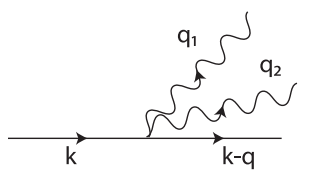

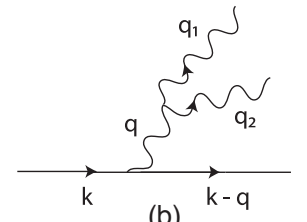

(b)

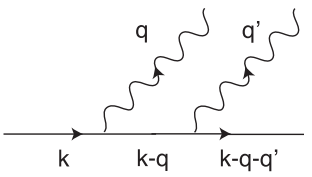

(d)
FIG. 3. Phonon creation by a dark matter particle. (a) Singlephonon creation. (b) Creation of a single phonon which transforms into two phonons (this process also describes direct creation of three phonons, one of which is absorbed by the dark matter particle). (c) Direct creation of two phonons at the dark matter- ${ }^{4} \mathrm{He}$ vertex. (d) Successive creation of two phonons by the dark matter particle. Process (d) is higher order in the dark matter-helium scattering length and can be ignored in estimating the event rate for a dark matter particle creating phonons.

$$
\frac{d \Gamma}{d q d \omega}=\Gamma_{0} \frac{q}{2 k^{2}} S(k, \omega),
$$

where $\Gamma_{0}=\sigma_{\chi_{4}} n_{\chi} n_{4} v$ is the rate of interaction per unit volume of the $\chi$ 's with a gas of noninteracting ${ }^{4} \mathrm{He}$ atoms at rest. In addition, the total rate for given momentum transfer $q$ is

$$
\frac{d \Gamma}{d q}=\frac{\Gamma_{0}}{2} \frac{q}{k^{2}} \int_{0}^{q v-q^{2} / 2 m_{\chi}} d \omega S(q, \omega) .
$$

\section{SUM-RULE CONSTRAINTS: SINGLE-PHONON VS MULTIEXCITATION EMISSION}

Although the momentum transfers from dark matter particles are below $\sim 0.7 \AA^{-1}$ for $m_{\chi}$ below $1 \mathrm{MeV}$ and $v \sim 230 \mathrm{~km} / \mathrm{s}$, energy transfers for momentum transfer $q$ can be much larger than $\omega(q)$, the energy required to generate a single phonon. However, for low $q$, the spectral weight of the structure function $S(q, \omega)$ is dominated by the single-phonon contribution, and the most likely outcome is that for dark matter in the sub-MeV mass range a single phonon will be produced. We can estimate the importance of multiexcitation processes from the $f$-sum rule [Eq. (10)] obeyed by $S(q, \omega)$, which at zero temperature has the form

$$
\int_{0}^{\infty} d \omega \omega S(q, \omega)=\frac{q^{2}}{2 m_{4}} .
$$

The sum rule follows directly from the relation between $S(q, \omega)$ and the density-density correlation function in the complex frequency plane, as we recall in Appendix A; this relation also provides the basis for expanding $S(q, \omega)$ at low $q$ in terms of phonon excitations. In Appendix B, we review the structure of $S(q, \omega)$ at finite temperature.

Neglecting the structure of the single-phonon peak for anomalous dispersion, as described below, one can write, for $\omega>0$,

$$
S(q, \omega)=Z(q) \delta\left(\omega-\omega_{q}\right)+S_{M}(q, \omega),
$$

where $Z(q)$ is the single-phonon weight, and $S_{M}(q, \omega)$ is the remaining multiexcitation strength.

The total excitation strength is bounded by the energy-weighted sum rule [Eq. (10)]. At zero temperature, in the absence of significant multiexcitation strength, $Z(q) \rightarrow q^{2} / 2 m_{4} \omega(q)$. With increasing $q$, the weight of the single-phonon peak in Eq. (11) is reduced from $q^{2} / 2 m_{4} \omega(q)$ by direct creation of two (or more) phonons and rotons; the sum-rule arguments given in Refs. [59,60] and reviewed in Ref. [53] indicate that 


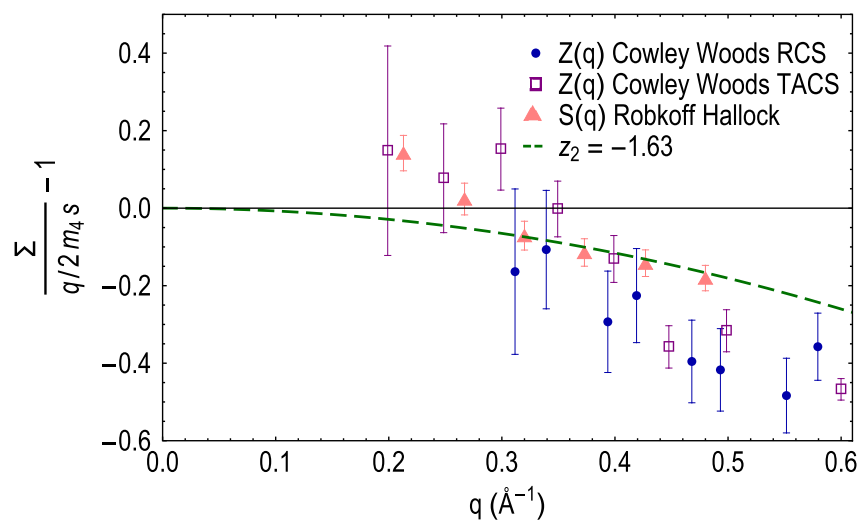

FIG. 4. Neutron scattering measurements of the relative deviations of the static structure function, $S(q)=\int d \omega S(q, \omega)$, and the single-phonon strength $Z(q)$ from their zero-temperature longwavelength single-phonon value, $q / 2 m_{4} s$. Here $\Sigma=S$ or $Z$. Points are from Robkoff and Hallock [61] at $T=1.38 \mathrm{~K}(\boldsymbol{\Delta})$, and from Cowley and Woods [62], at $T=1.1 \mathrm{~K}$, using the Rotating Crystal Spectrometer (RCS, •) and the Triple Axis Crystal Spectrometer (TACS, 口) at Chalk River. The data have been corrected to zero temperature by dividing the experimental $S_{T}(q)$ at finite $T$ by $1+2 n(\omega(q))$ and the experimental $Z(q)$ by $1+n(\omega(q))$, where $n(\omega(q))=\left(e^{\omega(q) / T}-1\right)^{-1}$ is the Bose occupation factor for excitations of momentum $q$. See the discussion in Appendix B. The dotted curve shows the sumrule-based fit [Eq. (12)].

$$
Z(q)=\frac{q^{2}}{2 m_{4} \omega(q)}\left(1-z_{2}\left(\frac{q}{m_{4} s}\right)^{2}+\cdots\right)
$$

where $z_{2} \simeq 1.63$, a value consistent with neutron scattering experiments [61-63]; see Eq. (B14) for the expansion to higher order in $q$. The correction $\sim z_{2}$ is shown as the dashed curve in Fig. 4.

The $f$-sum rule together with Eq. (12) then implies that for small $q$,

$$
\int_{0}^{\infty} d \omega \omega S_{M}(q, \omega)=z_{2}\left(\frac{q}{m_{4} s}\right)^{2} \frac{q^{2}}{2 m_{4}},
$$

plus terms of relative order $q^{6}$. Thus, multiexcitations contribute a fraction

$$
\int_{0}^{\infty} d \omega \omega S_{M}(q, \omega) / \int_{0}^{\infty} d \omega \omega S(q, \omega)=z_{2}\left(\frac{q}{m_{4} s}\right)^{2}
$$

to the sum rule at small $q$. At $q \lesssim 0.35 \AA^{-1}$, with $m_{4} s=1.50 \AA^{-1}$, the multiexcitation contribution is $\lesssim 10 \%$ of the single-phonon contribution.

Similarly, the static structure function is

$$
S(q)=\int d \omega S(q, \omega)=Z(q)+S_{M}(q),
$$

where the multiexcitation contribution is

$$
S_{M}(q)=\int_{0}^{\infty} d \omega S_{M}(q, \omega) .
$$

Since the multiexcitation weight is at $\omega \geq s q$, Eq. (13) implies that for small $q$,

$$
S_{M}(q) \leq \int_{0}^{\infty} d \omega \frac{\omega}{s q} S_{M}(q, \omega)=\frac{z_{2}}{2}\left(\frac{q}{m_{4} s}\right)^{3},
$$

or equivalently,

$$
\frac{S_{M}(q)}{S(q)} \leq z_{2}\left(\frac{q}{m_{4} s}\right)^{2}
$$

the same fraction as in the sum rule, Eq. (14).

The multiexcitation rate, $d \Gamma_{M} / d q$, for given $q$, compared to the single-phonon rate, is similarly bounded. We write

$$
\frac{d \Gamma}{d q}=\frac{d \Gamma_{1}}{d q}+\frac{d \Gamma_{M}}{d q}
$$

in terms of the single-phonon contribution

$$
\frac{d \Gamma_{1}}{d q}=\frac{\Gamma_{0}}{2} \frac{q}{k^{2}} Z(q) \simeq \frac{\Gamma_{0}}{4 m_{4} s} \frac{q^{2}}{k^{2}},
$$

where $q \leq 2 k$, and the multiexcitation contribution

$$
\frac{d \Gamma_{M}}{d q}=\frac{\Gamma_{0}}{2} \frac{q}{k^{2}} \int_{0}^{q v-q^{2} / 2 m_{\chi}} d \omega S_{M}(q, \omega) .
$$

Since the integral is bounded above by $S_{M}(q)$, we find from Eq. (B12) the bound on the multiexcitation rate,

$$
\frac{d \Gamma_{M}}{d q} \leq \frac{\Gamma_{0}}{4 k^{2}} \frac{z_{2} q^{4}}{\left(m_{4} s\right)^{3}} .
$$

Comparing with Eq. (20), we have

$$
\frac{d \Gamma_{M}}{d q} / \frac{d \Gamma_{1}}{d q} \leq z_{2}\left(\frac{q}{m_{4} s}\right)^{2},
$$

the same ratio as the contributions to the sum rule. The multiexcitation rate at $q \lesssim 0.4 \AA^{-1}$ is similarly $\lesssim 10 \%$ of the single-phonon contribution. The sum-rule argument implicitly takes into account the momentum dependence of the matrix elements for producing multiphonon states as well as the available phase space. We emphasize that this bound does not depend on whether the dispersion is anomalous or not.

When the phonon dispersion is normal single (on-shell) phonons cannot decay into two or more phonons. However, with anomalous dispersion a single phonon can decay into 
two, and there is no longer a clean distinction in $S(q, \omega)$ between damped single phonons and multiphonon states; the effect, as we see below, is to spread the single-phonon peak. Off-shell phonons can decay into two phonons if $\omega-\omega(q)>0$ for normal dispersion and if $\omega>2 \omega(q / 2)$, or equivalently $\omega-\omega(q)>-(\omega(q)-2 \omega(q / 2))$, for normal dispersion. For $\omega-\omega(q) \gg|\omega(q)-2 \omega(q / 2)|$, the decay rate becomes independent of the sign of the dispersion.

We turn to estimating the rate of single-phonon events. For simplicity, we assume here that the single-phonon spectrum cuts off at $q$ of the order of the ${ }^{4} \mathrm{He}$ Debye wave vector $k_{D}=1.089 \AA^{-1}$, defined in terms of the ${ }^{4} \mathrm{He}$ number density by $n_{4}=k_{D}^{3} / 6 \pi^{2}$. Equation (20) implies that the integrated one-phonon event rate, for a detector with a lower energy threshold $\omega_{0}$ with $\omega_{0} / s \leq 2 k=2 m_{\chi} v \leq k_{D}$, is

$$
\begin{aligned}
\Gamma_{1}\left(\omega>\omega_{0}\right) & =\int_{\omega_{0} / s}^{2 k} d q \frac{\Gamma_{0}}{m_{4} s} \frac{q^{2}}{4 k^{2}} \\
& =\frac{2 n_{4} \rho_{\chi} \sigma_{\chi_{4}}}{3 m_{4}} \frac{v^{2}}{s}\left(1-\left(\frac{\omega_{0}}{2 s m_{\chi} v}\right)^{3}\right),
\end{aligned}
$$

while in the limit of large $m_{\chi}$, with $\omega_{0} \leq k_{D} \leq 2 k$, one has

$$
\begin{aligned}
\Gamma_{1}\left(\omega>\omega_{0}\right) & =\int_{\omega_{0} / s}^{k_{D}} d q \frac{\Gamma_{0}}{m_{4} s} \frac{q^{2}}{4 k^{2}} \\
& =\frac{n_{4} \rho_{\chi} \sigma_{\chi_{4}}}{12 m_{4} s} \frac{k_{D}^{3}}{m_{\chi}^{3} v}\left(1-\left(\frac{\omega_{0}}{s k_{D}}\right)^{3}\right) .
\end{aligned}
$$

The prefactor of $\left(1-\left(\omega_{0} / 2 s m_{\chi} v\right)^{3}\right)$ in Eq. (24) is

$\simeq 3.0 \times 10^{-9}\left(\frac{\sigma_{\chi_{4}}}{10^{-40} \mathrm{~cm}^{2}}\right)\left(\frac{v}{230 \mathrm{~km} / \mathrm{s}}\right)^{2} \mathrm{~s}^{-1} \mathrm{~cm}^{-3}$,

where $\rho_{\chi}=n_{\chi} m_{\chi} \simeq 0.4 \mathrm{GeV} / \mathrm{cm}^{3}$ is the local dark matter density. The integrated rate for small $m_{\chi} v$ is proportional to $\rho_{x}$, and depends on $m_{\chi}$ only through the factor $\omega_{0} / 2 s m_{\chi} v$. The factor $2 s m_{\chi} v$ is $\sim 0.12\left(m_{\chi} / 100 \mathrm{keV}\right)$ $(v / 230 \mathrm{~km} / \mathrm{sec}) \mathrm{meV}$.

Figure 5 shows the one-phonon rate integrated over the standard halo model described above, $\int d v f(v) \Gamma_{1}\left(\omega>\omega_{0}\right)$, for various $m_{\chi}$. For low $m_{\chi}$, the zero-threshold rate scales with the mean square velocity $\int d v v^{2} f(v)=(369 \mathrm{~km} / \mathrm{s})^{2}$, and the single-phonon rate is given by

$\left\langle\Gamma_{1}(\omega>0)\right\rangle \sim 8.9 \times 10^{-9} \mathrm{~cm}^{-3} \mathrm{~s}^{-1}\left(\sigma_{\chi_{4}} / 10^{-40} \mathrm{~cm}^{2}\right)$.

Note that the phase space for creating single phonons falls rapidly for $m_{\chi} \gtrsim 500 \mathrm{keV}$, as seen in Fig. 5 .

More generally, the contribution of the single-phonon peak to the interaction rate of a dark matter particle with the helium [with $Z(q) \rightarrow q^{2} / 2 m_{4} \omega(q)$ ] is

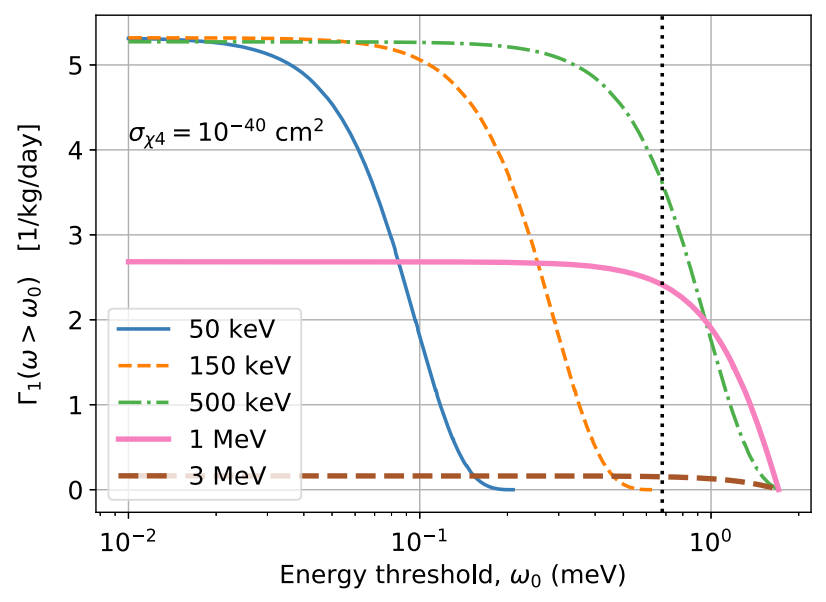

FIG. 5. Rate of single-phonon events per $\mathrm{kg}$ of ${ }^{4} \mathrm{He}$ for $\sigma_{\chi 4}=$ $10^{-40} \mathrm{~cm}^{2}$ and a selection of masses $m_{\chi}$, as a function of limiting energy threshold $\omega_{0}$. Rates are integrals of $\Gamma_{1}\left(\omega>\omega_{0}\right)$ [Eqs. (24) and (25)] over the standard halo model, following the parameters in Sec. II A. The vertical dotted line indicates $\omega\left(q_{c}\right)$ at 1 bar, below which phonons can split into two or more phonons. The rapid falloff for $\mathrm{MeV}$-scale $m_{\chi}$ arises from the lack of phase space for single-phonon production.

$$
\frac{d \Gamma_{1}}{d q d \cos \theta_{q}}=\frac{\Gamma_{0}}{4 m_{\chi}^{2} v} \frac{q^{4}}{m_{4} \omega(q)} \delta(\omega-\omega(q)) .
$$

Integrating over $q$ using Eq. (4), we find the angular distribution,

$$
\frac{d \Gamma_{1}}{d \cos \theta_{q}} \simeq \frac{2 m_{\chi} \Gamma_{0}}{v m_{4} s}\left(v \cos \theta_{q}-s\right)^{2},
$$

with the restriction that $0<v \cos \theta_{q}-s \lesssim k_{D} / 2 m_{\chi}$.

Figure 6 shows the one-phonon rate, Eq. (29), as a function of $\cos \theta_{q}$, assuming a cross section, $\sigma_{\chi_{4}}=$ $10^{-40} \mathrm{~cm}^{2}$, and a nominal velocity $v=230 \mathrm{~km} / \mathrm{s}$, and $2 m_{\chi} v \lesssim k_{D}$.

Measurement of the momentum and energy transfers $\vec{q}$ and $\omega$ from detection of either single-phonon or phononpair production, implies that the mass of the dark matter particle is

$$
m_{\chi}=\frac{q^{2}}{2\left(q v \cos \theta_{q}-\omega\right)} .
$$

For single-phonon detection, $\omega \simeq s q$, and thus

$$
m_{\chi}=\frac{q}{2\left(v \cos \theta_{q}-s\right)} .
$$

Except for emission at $\theta_{q} \simeq \pi / 2$, one can neglect the $s$ and conclude that $m_{\chi} \simeq 1.36 q / \cos \theta_{q}$, where $m_{\chi}$ is measured in $\mathrm{MeV}$ and $q$ in $\AA^{-1}$. 


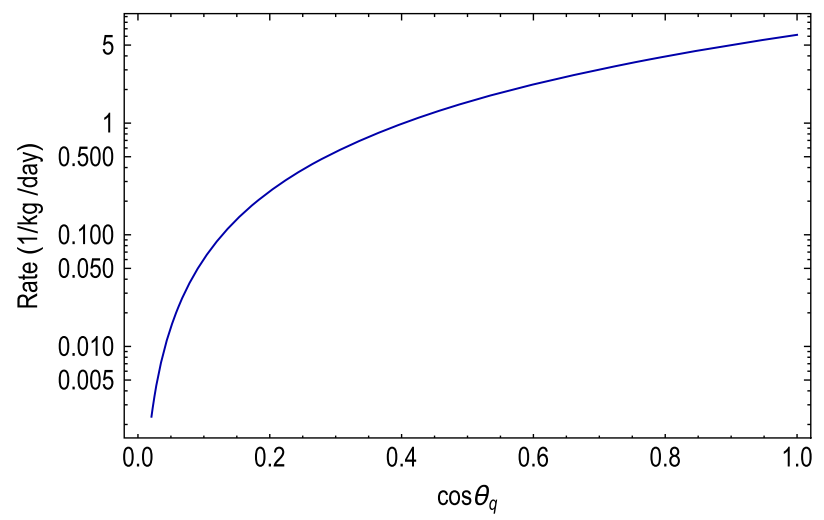

FIG. 6. Event rate for dark matter particles producing single phonons in the normal dispersion region, in units of events $/ \mathrm{kg} /$ day. The dark matter-helium cross section is taken to be $\sigma_{\chi_{4}}=10^{-40} \mathrm{~cm}^{2}, v=230 \mathrm{~km} / \mathrm{s}$, and the detector acceptance to be $\Delta \cos \theta_{q}=0.1$. Kinematics requires $v \cos \theta_{q}$ to lie in the interval $s$ to $s+k_{D} / 2 m_{\chi}$.

\section{PHONON DAMPING}

The principal damping mechanism of a phonon, either on or off shell, is decay into two phonons when kinematically allowed. A single phonon produced on shell will decay into two phonons as long as its momentum is less than the critical $q_{c}\left[=0.4215 \AA^{-1}\right.$ for the dispersion relation (2)]. If $q>q_{c}$, the phonon is stable. However, the production of two phonons via the process in Fig. 3(b) remains possible for all $q$ if the single phonon is sufficiently off shell.

To understand the splitting of an on-shell phonon of momentum $q$ into a pair of phonons of momenta $q_{1}$ and $q_{2}$ (where in this discussion we assume $q_{1} \leq q_{2}$ ), we note that the dispersion curve (2) has a number of critical points. Beyond the inflection point, where $\omega^{\prime \prime}\left(q_{\text {infl }}\right)=0$, at $q_{\text {infl }}=0.216 \AA^{-1}$, the slope of the dispersion curve equals the zero-momentum sound speed $s$ at $q_{s}=0.377 \AA^{-1}$; up to $q_{s}$, a phonon of momentum $q$ can turn into a pair of phonons, where the smaller of the momenta $q_{1}$ can be arbitrarily small. The critical momentum $q_{c}=0.422 \AA^{-1}$ is where $\omega\left(q_{c}\right)=2 \omega\left(q_{c} / 2\right)$, so that one on-shell phonon can decay into two collinear equal-momenta phonons. Phonons of momentum beyond $q_{c}$ can no longer split into pairs of phonons. Between $q_{s}$ and $q_{c}$, phonons can still turn into pairs, but with both $q_{1}$ and $q_{2}$ finite. Up to $q_{c}, q_{1}$ takes on its maximum possible value when $q_{1}=q_{2} \gtrsim q / 2$, with equality at $q=q_{c}$. Lastly, we note that $\omega(q)=s q$ again at $q=q_{a}=0.542 \AA^{-1}$.

In the decay of a phonon of momentum $q$ into $q_{1}$ and $q_{2}$, (again with $q_{1} \leq q_{2}$, so that one does not have symmetry under $q_{1} \leftrightarrow q_{2}$ ), the phonons are collinear only if $q_{1}=q_{1, \min }$. For $q_{1}>q_{1, \min }$, the two phonons are at a finite angle with respect to $\vec{q}$. At $q_{1}=q_{1, \max }$, one has $q_{1}=q_{2}$. The range of $q_{1}$ in the splitting of an on-shell
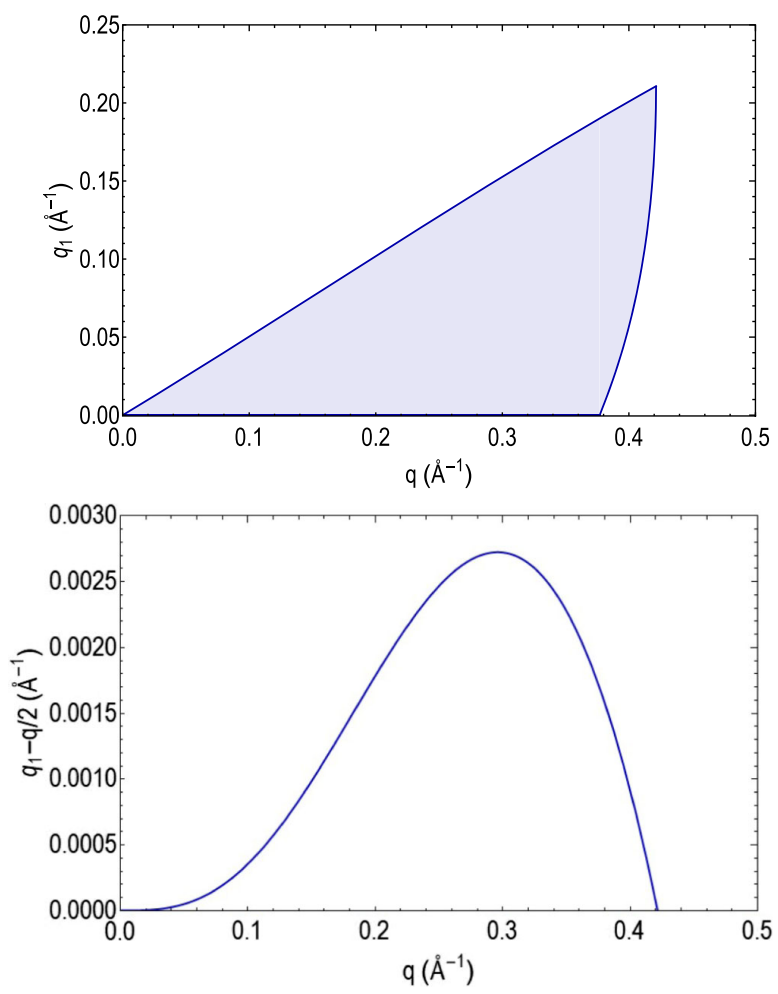

FIG. 7. The range of $q_{1}$, the smaller of $q_{1}$ and $q_{2}$ in the single splitting of an on-shell phonon of momentum $q$ into phonons $\vec{q}_{1}$ and $\vec{q}_{2}$, as a function of $q$, is shown as the shaded region. The lower panel shows the fine structure of the upper limit $q_{1}$ compared with $q / 2$, on a scale $\sim 0.01$ of that in the upper panel.

phonon of momentum $q$, as a function of $q$, is shown in the upper panel of Fig. 7. Owing to the smallness of the anomalous dispersion, the upper limit is only slightly above $q / 2$, as shown in the lower panel of Fig. 7 .

We now examine the rate of decay $\gamma_{2}(q, \omega)$ of a phonon of momentum $q$ and (possibly off-shell) energy $\omega \geq \omega(q)$ into two phonons. In a system of finite volume $\Omega$ (which we take to infinity in the end), the matrix element for a phonon of momentum $\vec{q}$ to generate a pair of phonons $\vec{q}_{1}$ and $\vec{q}_{2}$ is $\left\langle\vec{q}_{1}, \vec{q}_{2}|V| \vec{q}\right\rangle \delta_{\vec{q}_{1}+\vec{q}_{2}, \vec{q}} / \sqrt{\Omega}[64]$, where

$$
\begin{aligned}
\left\langle\vec{q}_{1}, \vec{q}_{2}|V| \vec{q}\right\rangle= & \sqrt{\frac{q^{2} q_{1}^{2} q_{2}^{2} s^{4}}{8 m_{4} n_{4} \omega(q) \omega\left(q_{1}\right) \omega\left(q_{2}\right)}} \\
& \left.\times\left(2 u-1+\hat{q}_{1} \cdot \hat{q}_{2}+\left(\hat{q}_{1}+\hat{q}_{2}\right) \cdot \hat{q}\right)\right),
\end{aligned}
$$

with $u=\left(n_{4} / s\right) \partial s / \partial n_{4}$ as the phonon Grüneisen parameter, $\approx 2.843$ at SVP. The latter angular term is bounded above by $2(u+1)$ and below by $2(u-1)$. In the following, rather than going through a complicated calculation involving the phonon angles, we replace this factor with $2(u+\nu)$, where for back-to-back phonons, $\nu=-1$, and for collinear phonons, $\nu=1$. The rate at which a phonon of momentum 
$q$ and energy $\omega$ decays into two phonons of momenta $\vec{q}_{1}$ and $\vec{q}_{2}$ is

$$
\begin{aligned}
\gamma_{2}(q, \omega)= & \frac{1}{2} \int \frac{d^{3} q_{1}}{(2 \pi)^{3}} \frac{d^{3} q_{2}}{(2 \pi)^{3}}\left|\left\langle\vec{q}_{1}, \vec{q}_{2}|V| \vec{q}\right\rangle\right|^{2} \\
& \times(2 \pi)^{4} \delta\left(\omega-\omega\left(q_{1}\right)-\omega\left(q_{2}\right)\right) \delta\left(\vec{q}-\vec{q}_{1}-\vec{q}_{2}\right) .
\end{aligned}
$$

The factor $1 / 2$ compensates for double-counting the twophonon states (since $\left|\vec{q}_{1} \vec{q}_{2}\right\rangle=\left|\vec{q}_{2} \vec{q}_{1}\right\rangle$ ). In the calculation below, the curvature of the phonon spectrum is important in the energy-conserving delta function, but not in the matrix elements; thus, we replace the various $\omega\left(q_{i}\right)$ 's in the prefactors in Eq. (32) with their values for linear dispersion, $s q_{i}$.

To evaluate the integral in Eq. (33), we use momentum conservation to do the $q_{2}$ integral, so that $\vec{q}_{2} \rightarrow \vec{q}-\vec{q}_{1}$, and we find

$$
\begin{aligned}
\gamma_{2}(q, \omega)= & \frac{(u+\nu)^{2}}{m_{4} n_{4}} \int \frac{d q_{1}}{8 \pi} d \cos \delta_{1} q_{1}^{3} q_{2} \\
& \times \delta\left(\omega-\omega\left(q_{1}\right)-\omega\left(q_{2}\right)\right),
\end{aligned}
$$

where $\delta_{1}$ is the angle between $\vec{q}$ and $\vec{q}_{1}$. The relation $q_{2}^{2}=q^{2}+q_{1}^{2}-2 q q_{1} \cos \delta_{1}$ implies that

$$
\int d \cos \delta_{1} \delta\left(\omega-\omega\left(q_{1}\right)-\omega\left(q_{2}\right)\right)=\frac{q_{2}}{q q_{1} d \omega\left(q_{2}\right) / d q_{2}},
$$

where $\Theta$ is unity if there is an angle $\delta_{1}$ for which the argument of the energy delta function vanishes, and zero otherwise; hence,

$$
\begin{aligned}
\gamma_{2}(q, \omega) & =\frac{(u+\nu)^{2} s}{8 \pi m_{4} n_{4}} \int d q_{1} \frac{q_{1}^{2} q_{2}^{2}}{\partial \omega_{2} / \partial q_{2}} \\
& =\frac{(u+\nu)^{2} s \omega}{8 \pi m_{4} n_{4}} \int_{0}^{1} d x \frac{q_{1}^{2} q_{2}^{2} \Theta}{d \omega_{1} / d q_{1} d \omega_{2} / d q_{2}} .
\end{aligned}
$$

In the lower equation, we integrate with respect to $x \equiv$ $\omega_{1} / \omega$ at fixed $\omega$. Note that $\omega_{2} / \omega=1-x$. The range of $q_{1}$ is determined by the requirement that there be an angle $\delta_{1}$ for which the argument of the energy delta function vanishes, and $\Theta$ includes the same restrictions in terms of $x$.

Were the dispersion normal, the decay of one phonon to two could only take place for $\omega>\omega(q)$. We explicitly evaluate the integral in Eq. (36) for small $q$ and $\omega-\omega(q) \ll s q$, using the dispersion relation in the form of Eq. (3). For given $\omega-\omega(q)$, the minimum $q_{1}$ is achieved for $\vec{q}_{1}$ antiparallel to $\vec{q}$, and from energy conservation, $q_{1}^{\min } \approx(\omega-\omega(q)) / 2 s$. On the other hand, the maximum $q_{1}$ is achieved for $\vec{q}_{1}$ parallel to $\vec{q}$; again from energy conservation, $q_{1}^{\max } \approx 3(\omega-\omega(q)) / s \zeta_{N} q^{2}$. For small $q$ in both limits, $q_{2} \approx q$, and the integral in Eq. (36) is approximately $\left(q_{1}^{\max }\right)^{3} / 3 s$. Altogether, then,

$$
\gamma_{2}(q, \omega)=(u+1)^{2} \frac{\pi q^{2}}{2 m_{4}}\left(\frac{\omega-\omega(q)}{s k_{D} \kappa_{N}}\right)^{3} \equiv \mathcal{C}(\omega-\omega(q))^{3}
$$

for $\omega \gtrsim \omega(q)$; here $\kappa_{N} \equiv 3 \zeta_{N} q^{2}$; at SVP, $\kappa_{N}=0.98\left(q / k_{D}\right)^{2}$. In deriving Eq. (38), we have replaced $\nu$ with +1 since for small $q$ the integral is dominated by collinear phonons. The damping vanishes at $\omega=\omega(q)$, as expected. For $\omega$ sufficiently close to $\omega(q), S$ [from Eq. (A7) with $\mathcal{R}$ neglected] reduces to a delta function

$$
S(q, \omega) \rightarrow \frac{q^{2}}{2 m_{4} \omega(q)} \delta(\omega-\omega(q))
$$

plus a continuum, corresponding to multiphonon excitations, which, as is schematically illustrated in Fig. 8, goes to zero as $\omega-\omega(q)$ when $\omega \rightarrow \omega(q)$ from above [see Eq. (46) below].

On the other hand, the direct two-phonon contribution to $S_{2}(q, \omega)$, determined by the matrix element (51) below, has the same structure in $\omega$ as $\gamma_{2}(q, \omega)$, and is of the order $(\omega-\omega(q))^{3}$. The delta function, Eq. (39), corresponds to a sharp single-phonon line, which exhausts the $f$-sum rule [Eq. (10)]. In the $f$-sum rule, the integral over the multiphonon background is canceled by renormalization corrections to the delta function from the real part of the phonon self-energy, $\mathcal{R}$, neither of which we consider here.

With a purely linear spectrum, decay of one phonon into two is allowed for all energies greater than $s q$ and forbidden

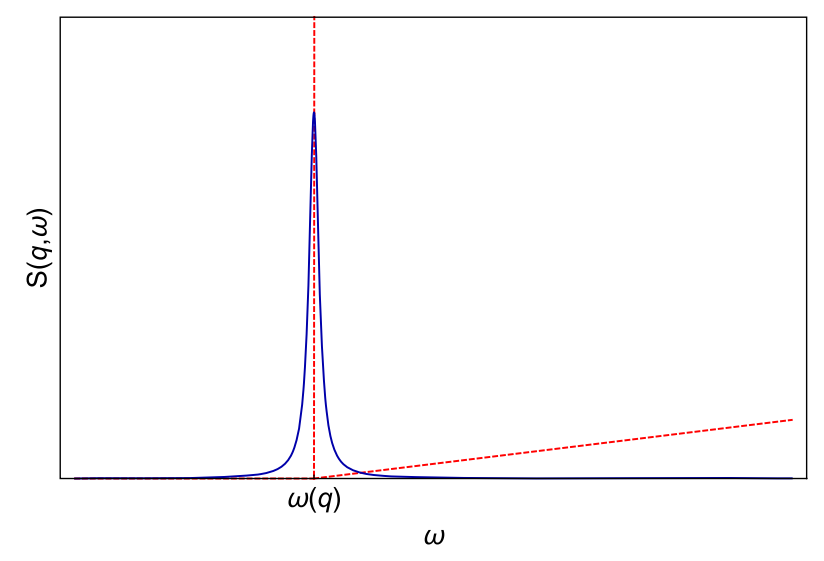

FIG. 8. Schematic behavior of $S(q, \omega)$ near $\omega(q)$. At higher $q$, where the phonon dispersion is normal, (dashed), $S$, has a zerowidth delta function at $\omega=\omega(q)$ and a multiphonon contribution initially linearly increasing for $\omega>\omega(q)$ [Eq. (46)], while at lower $q$, where the dispersion is anomalous (solid), the delta function is spread out; see Eq. (40). 
for energies less than $s q$, so $\gamma_{2}(\omega, q)$ is discontinuous at $\omega=s q$.

For anomalous dispersion with $q \leq q_{s}$, all lowmomentum on-shell phonons can decay, and the range of the $x$ integral in Eq. (37) is 0 to 1 . For small $q$, and $0 \leq \omega-\omega(q) \ll s q$, we find

$$
\gamma_{2}(q, \omega)=(u+1)^{2} \frac{\pi q^{5}}{40 m_{4} k_{D}^{3}} \equiv \frac{q^{5} s}{\eta} .
$$

As $\omega$ decreases below $\omega(q)$, decay is still possible, with $\gamma_{2}$ vanishing for $\omega \leq 2 \omega(q / 2)$, as illustrated in Fig. 8 .

While in this calculation we assume anomalous dispersion in the simplified form [Eq. (1)], this result is independent of the details of the anomalous dispersion, as long as it is small, and holds equally well for the more accurate dispersion relation [Eq. (2)].

The corresponding phonon mean free path as limited by decay into two phonons is

$$
\ell(q)=\frac{s}{\gamma_{2}}=\frac{\eta}{q^{5}}=\frac{1.67 \AA}{q^{5}},
$$

with $q$ measured in $\AA^{-1}$ in the final expression. ${ }^{4,5}$

Near $\omega=\omega(q), S(q, \omega)$ for anomalous dispersion is essentially Lorentzian; the spread in the peak at $\omega=\omega(q)$ is found by noting that the phonon peak is effectively shifted to

$$
\omega=\omega(q)-\frac{i}{2} \gamma_{2}(q, \omega(q))
$$

\footnotetext{
${ }^{4}$ Similarly, the rate of absorption of a phonon on a thermal phonon (the Landau process of two phonons to one) is given by Eq. (40), only with $q^{5}$ replaced by $q(2 \pi T / s)^{4}$ for $q \ll T / s$ $[34,65,66]$. For phonon energies large compared with those of thermal phonons, the absorption rate varies as $q^{2}(T / s)^{3}$, and this process can be ignored.

${ }^{5}$ This mean free path is the length for a single phonon of momentum $\vec{q}$ in liquid helium to decay into two almost collinear phonons when no other excitations are present initially. When many phonons are present, this mean free path determines the rate at which phonons moving in approximately the same direction come into thermal equilibrium with each other due to one phonon decaying into two, and the inverse process in which two almost collinear phonons create a single phonon. The characteristic mean free paths for thermal conductivity or viscosity $[51,67]$ are very much larger because processes involving nearly collinear phonons are ineffective in degrading heat currents or stresses: to dampen these disturbances requires establishing equilibrium between phonons moving in directions differing by angles $\sim 1$ radian, and this can only be done by a sequence of decays and coalescences that equilibrate phonons moving in slightly different directions $[35,68]$. The difference between $\ell$ and the mean free path for thermal conduction or viscosity is similar to the difference between the total cross section for scattering of a particle and the transport cross section in the kinetic theory of gases and the theory of impurity resistivity in metals [69].
}

The peak, which is relatively narrow, again exhausts the $f$-sum rule, with contributions of the tail of $S$ to the sum rule being canceled by renormalization corrections; see Appendix A. The Beliaev process spreads what would be a sharp single-phonon peak at $\omega=\omega(q)$ into the two-phonon continuum.

The single-phonon peak in $S(q, \omega)$ for anomalous dispersion does not cut off abruptly with decreasing $\omega$ at $\omega=\omega(q)$. Rather, with $\omega$ increasing from zero, the first possible decay of a phonon of momentum $q$ is into two equal-momentum phonons; thus, the minimum $\omega$ at which $\gamma_{2}$ is nonzero is $\omega_{\min }=2 \omega(q / 2)$, or

$$
\omega_{\min }=\omega(q)-\frac{3}{4} s \zeta_{A} q^{3}
$$

The structure of $S$ near $\omega=\omega(q)$ for anomalous as well as normal dispersion is shown in Fig. 8.

The rate of excitation of ${ }^{4} \mathrm{He}$ via the process in Fig. 3(b) (denoted by the subscript $b$ ) is

$$
d \Gamma_{b}=n_{\chi}\left(\frac{2 \pi a}{m_{r}}\right)^{2} 2 \pi n_{4} S_{b}(q, \omega) \frac{d^{3} q}{(2 \pi)^{3}}
$$

where

$S_{b}(q, \omega)=\frac{q^{2}}{\pi m_{4}} \frac{\omega(q) \gamma_{2}(q, \omega)}{\left(\omega^{2}-\omega(q)^{2}\right)^{2}+\omega(q)^{2} \gamma_{2}(q, \omega)^{2}}$

is the structure function, as derived in Appendix A, with only the contribution from two-phonon states in $\gamma(q, \omega)$ included, and self-energy effects $\mathcal{R}$ in the denominator of Eq. (A7) neglected. With $\gamma_{2}$ from Eq. (40), we find that the relative half-width of the single phonon peak at half height is $|\omega-\omega(q)| / \omega(q) \sim q^{4} / 2 \eta$, which is $\sim 0.3 q^{4}$ with $q$ measured in inverse angstroms; the peak is very narrow. In the limit in which the width $\gamma_{2}$ goes to $0, S_{b}(q, \omega)$ becomes simply the one-phonon structure function, Eq. (39).

For normal dispersion, Eq. (45), with Eq. (38), implies that for $\omega$ just above $\omega(q)$,

$$
S_{b}(q, \omega) \simeq \frac{q^{2} \mathcal{C}}{4 \pi m_{4} \omega(q)}(\omega-\omega(q))
$$

We note that for anomalous dispersion for small $q$, the integrand in Eq. (37) gives the relative probability of the initial phonon $q$ decaying to phonons with momenta $q_{1}$ and $q_{2}$. The normalized probability for small $q$ is

$$
\frac{d P}{d x}=30 x^{2}(1-x)^{2},
$$

where $0 \leq x \leq 1$. 


\section{TWO-PHONON EMISSION}

Production of multiple phonons proceeds by the creation of an initial off-shell phonon which converts into multiphonon states, the process in Fig. 3(b), or else by direct production of a multiphonon state by the dark matter particle, the process in Fig. 3(c). These two processes are coherent. The emission of two phonons via an intermediate single phonon has the amplitude

$$
A_{2}^{1}=\frac{2 \pi a}{m_{\chi}}\left\langle\vec{q}_{1}, \vec{q}_{2}|\rho| 0\right\rangle_{\text {one phonon }}
$$

times $\delta_{\vec{q}_{1}+\vec{q}_{2}, \vec{q}} / \sqrt{\Omega}$, where $|0\rangle$ is the ${ }^{4} \mathrm{He}$ ground state, and

$$
\begin{aligned}
& \left\langle\vec{q}_{1}, \vec{q}_{2}|\rho| 0\right\rangle_{\text {one phonon }} \\
& \quad=\left(\frac{q^{2} n_{4}}{2 m_{4} \omega(q)}\right)^{1 / 2} \frac{2 \omega(q)\left\langle\vec{q}_{1}, \vec{q}_{2}|V| \vec{q}\right\rangle}{\omega^{2}-\omega(q)^{2}+i \omega(q) \gamma(q, \omega)} .
\end{aligned}
$$

The factor $\left(q^{2} n_{4} / 2 m_{4} \omega(q)\right)^{1 / 2}$ is the amplitude for the density operator to create a phonon of momentum $q$; the following factor corresponds to the combination of the energy denominators $(\omega-\omega(q)+i \gamma(q, \omega) / 2)^{-1}-$ $(\omega+\omega(q)+i \gamma(q, \omega) / 2)^{-1}$ describing the propagation of the phonon.

The direct production of two phonons by the dark matter particle, Fig. 3(c), has the amplitude

$$
A_{2}^{d}=\frac{2 \pi a}{m_{\chi}}\left\langle\vec{q}_{1}, \vec{q}_{2}|\rho| 0\right\rangle_{\text {direct }}
$$

times $\delta_{\vec{q}_{1}+\vec{q}_{2}, \vec{q}} / \sqrt{\Omega}$. While the amplitude for the density operator to create two phonons directly is not well determined over the relevant range of momenta, its leading dependence at low $q$ is $\sim q^{2}$; this result has previously been demonstrated for specific models [29,37,38], but, as we show in Appendix C, it is a straightforward consequence of translational invariance. Thus, we write

$$
\left\langle\vec{q}_{1}, \vec{q}_{2}|\rho| 0\right\rangle_{\mathrm{direct}} \equiv \frac{q^{2}}{m_{4}} \frac{\mathcal{M}}{s k_{D}} .
$$

We estimate that its strength is $\mathcal{M} \sim 1$ from sum-rule arguments in Appendix C.

The total matrix element for creating two phonons, the sum of Eqs. (49) and (51), takes the form $\left(q^{2} / m_{4}\right)$ $\left\langle\vec{q}_{1}, \vec{q}_{2}|M| 0\right\rangle$, where with linear phonon dispersion,

$\left\langle\vec{q}_{1}, \vec{q}_{2}|M| 0\right\rangle \equiv \frac{s \sqrt{q_{1} q_{2}}(u+\nu)}{\omega^{2}-\omega(q)^{2}+i \omega(q) \gamma_{2}(q, \omega)}+\frac{\mathcal{M}}{s k_{D}}$.

Near the resonance $[\omega \simeq \omega(q)$ in the denominator], the one-to-two phonon process is dominant. However, for $q_{1}, q_{2} \sim k_{D}$ away from resonance, the two amplitudes $A_{2}^{1}$ and $A_{2}^{d}$ are comparable in magnitude.

The differential rate of two-phonon emission per unit volume of ${ }^{4} \mathrm{He}$ is

$$
\begin{aligned}
d \Gamma_{2} \simeq & \frac{\pi \Gamma_{0}}{2 m_{\chi} k n_{4}} \int \frac{d^{3} q_{1}}{(2 \pi)^{3}} \frac{d^{3} q_{2}}{(2 \pi)^{3}} \frac{q^{4}}{m_{4}^{2}}\left|\left\langle\vec{q}_{1}, \vec{q}_{2}|M| 0\right\rangle\right|^{2} \\
& \times 2 \pi \delta\left(\omega-\omega\left(q_{1}\right)-\omega\left(q_{2}\right)\right),
\end{aligned}
$$

where the factor $1 / 2$ again compensates for doublecounting of phonon final states; here $\vec{q}=\vec{q}_{1}+\vec{q}_{2}$. Comparing Eq. (53) with Eq. (6), we see that the dynamic structure factor for two-phonon states is

$$
\begin{aligned}
S_{2}(q, \omega)= & \frac{3 q^{4}}{8 \pi k_{D}^{3} m_{4}^{2}} \int d^{3} q_{1} d^{3} q_{2} \delta\left(\omega-\omega\left(q_{1}\right)-\omega\left(q_{2}\right)\right) \\
& \times \delta\left(\vec{q}-\vec{q}_{1}-\vec{q}_{2}\right)\left|\left\langle\vec{q}_{1}, \vec{q}_{2}|M| 0\right\rangle\right|^{2}
\end{aligned}
$$

We first evaluate $S_{2}(q, \omega)$ for $\omega$ large compared with $\omega(q)$. With the momentum delta function used to eliminate the $q_{2}$ integral, the $\cos \delta_{1}$ integral, as in Eq. (35), gives a factor $q_{2} / q q_{1} s$, so that

$S_{2}(q, \omega)=\frac{3 q^{3}}{4 k_{D}^{3} m_{4}^{2} s} \int_{0}^{\omega / s} d q_{1} q_{1} q_{2}\left|\left\langle\vec{q}_{1}, \vec{q}_{2}|M| 0\right\rangle\right|^{2}$.

Doing the $q_{1}$ integral with $q_{1}=\omega x$ and $q_{2}=(1-x) \omega$ as above, we find

$$
\begin{aligned}
S_{2}(q, \omega)= & \frac{q^{3}}{8 k_{D}^{3}} \frac{\omega}{\left(m_{4} s^{2}\right)^{2}} \\
& \times\left(\left(\frac{\omega \mathcal{M}}{s k_{D}}\right)^{2}+\frac{9 \pi}{32} \frac{\omega \mathcal{M}}{s k_{D}}(u-1)+\frac{(u-1)^{2}}{5}\right),
\end{aligned}
$$

where we let $\nu \rightarrow-1$ for approximately back-to-back phonons. In the regime of $\omega$ that can produce two phonons, $S(q, \omega)$ has approximately linear, quadratic, and cubic terms in $\omega$.

The rate of two-phonon emission in this regime is given by Eq. (9) with Eq. (56), with the upper limit on the $\omega$ integral essentially $2 s k_{D}$. Thus,

$$
\begin{aligned}
\frac{d \Gamma_{2}}{d q} & =\frac{\Gamma_{0} q^{4}}{4 k^{2} k_{D}\left(m_{4} s\right)^{2}}\left(\mathcal{M}^{2}+\frac{3 \pi}{16} \mathcal{M}(u-1)+\frac{(u-1)^{2}}{10}\right) \\
& \sim \frac{r q^{2}}{k_{D} m_{4} s} \frac{d \Gamma_{1}}{d q}
\end{aligned}
$$

where the two values $r=0.7$ and 0.9 reflect the two allowed values of $\mathcal{M}$. This estimate is consistent with the sum-rule result [Eq. (23)]. 
Integrating over $q$ from 0 to $k_{D}$ and averaging the velocity $v$ using the halo model as above, we find that the total zero-threshold two-phonon rate is given by

$$
\left\langle\Gamma_{2}(\omega>0)\right\rangle=\frac{8 r}{5} \frac{\sigma_{\chi_{4}} n_{4} \rho_{\chi}}{k_{D}\left(m_{4} s\right)^{2}} m_{\chi}^{2} I
$$

where $I=\int d v v^{4} f(v) \simeq(409 \mathrm{~km} / \mathrm{s})^{4}$. Numerically,

$$
\left\langle\Gamma_{2}(\omega>0)\right\rangle \sim 7.7 r \times 10^{-9} \mathrm{~cm}^{-3} \mathrm{~s}^{-1} \frac{\sigma_{\chi_{4}}}{10^{-40} \mathrm{~cm}^{2}}\left(\frac{m_{\chi}}{1 \mathrm{MeV}}\right)^{2}
$$

The two-phonon rate is less than $1 \%$ of the one-phonon rate for $m_{\chi} \lesssim 120 \mathrm{keV}$; the two averaged rates are approximately equal for $m_{\chi} \sim 1 \mathrm{MeV}$.

\section{PHONON CASCADES IN THE ANOMALOUS DISPERSION REGIME}

We next describe the behavior of individual phonons produced in superfluid ${ }^{4} \mathrm{He}$ by a dark matter particle. Owing to anomalous dispersion, phonons up to the critical momentum, $q_{c}$, decay rapidly into two phonons (the Beliaev process). The lifetime of a single on-shell phonon of momentum $q$ to decay into a pair of phonons is given by Eq. (40), and the corresponding phonon mean free path is given in Eq. (41). As a consequence of this rapid decay, a phonon of momentum below $q_{c}$ will generate a cascade of lower-momentum phonons, as illustrated in Fig. 9. Appendix $\mathrm{C}$ gives an extended description of the decay of a single phonon into two.

We first derive the opening angle in the three-phonon process, $\vec{q}_{1} \rightarrow \vec{q}_{3}+\vec{q}_{4}$. For simplicity, we present the calculation only in terms of the simplified anomalous dispersion relation, $\omega(q) \simeq s q\left(1+\zeta_{A} q^{2}\right)$. Since the deviations from linear dispersion in the anomalous dispersion regime are small, the angles $\delta_{3}$ and $\delta_{4}$ of the daughter phonons with respect to $\vec{q}_{1}$ are also small. Using $q_{4}^{2}=\left(\vec{q}_{1}-\vec{q}_{3}\right)^{2}=q_{1}^{2}+q_{3}^{2}-2 q_{1} q_{3} \cos \delta_{3}$, in the energy conservation condition, $\omega_{3}+\omega_{4}=\omega_{1}$, we find that to leading order (neglecting small terms $\sim \zeta_{A} q_{1}^{2} \delta_{3}^{2}$, etc.),

$$
\delta_{3}^{2} \simeq 6 \zeta_{A} q_{4}^{2}, \quad \delta_{4}^{2} \simeq 6 \zeta_{A} q_{3}^{2}
$$

The mean $\left\langle\delta_{3}^{2}\right\rangle$ of the phonon $\vec{q}_{3}$ is given by the integral of Eq. (60) weighted by the probability [Eq. (47)] that the daughter phonon carries a fraction $x$ of the original phonon energy:

$$
\left\langle\delta_{3}^{2}\right\rangle=\int_{0}^{1} d x 6 \zeta_{A} q_{1}^{2}(1-x)^{2} \frac{d P(x)}{d x}=\frac{12}{7} \zeta_{A} q_{1}^{2} .
$$

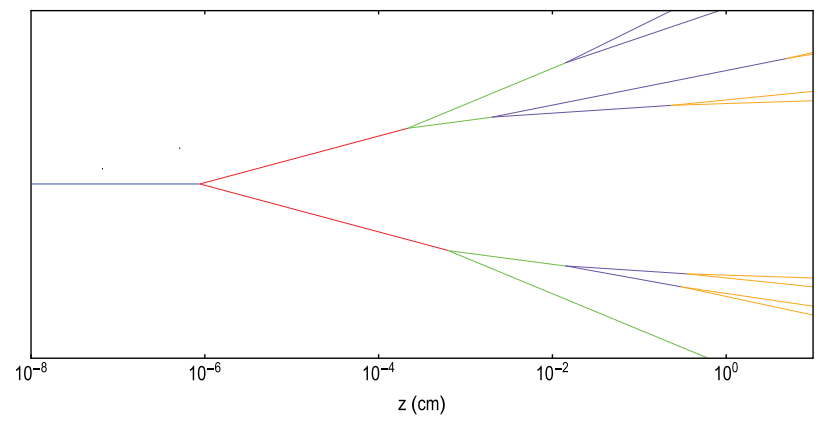

FIG. 9. Schematic drawing of a cascade of phonons in the anomalous dispersion region. The initial phonon energy is $0.5 \mathrm{meV}$ ( $q=0.32 \AA^{-1}$ ); the average phonon energy in the third (purple) generation is about $0.06 \mathrm{meV}$, where the mean free path is of order $\mathrm{cm}$. Path lengths and energy splittings in the figure correspond to Eqs. (41) and (47), respectively. The horizontal scale is logarithmic, and the vertical scale is essentially the distance from the extension of the path of the initial phonon, divided by $z$. For illustration, the paths are drawn as straight lines.

In a dark matter experiment, an initial phonon of momentum $\sim 0.1 \AA^{-1}$ in its first decay into two phonons would lead to an rms opening angle $\simeq 7.9^{\circ}$ between the initial phonon and each daughter. ${ }^{6}$

To see the general structure of the cascade of an initial phonon of momentum $q_{0}$ that is large compared with the momentum of thermal phonons, $\sim T / s$, at the ambient temperature $T$, we make the simplifying assumption that in each decay process a single phonon of momentum $q_{i}$ divides into two phonons each of momentum $q_{i} / 2$. [This is a reasonable approximation, given the peaking of the probability (47) about $x=1 / 2$; the mean square deviation from $1 / 2$ is $\left\langle(x-1 / 2)^{2}\right\rangle=1 / 28$.] The phonons will be at a slight angle with respect to each other, as calculated above. Were the mean free path a constant, $\ell_{0}$, the number of phonons $N(z)$ present at a distance $z$ from the initial phonon production point would be $N(z) \simeq 2^{z / \ell_{0}}$. The mean free path depends, however, on $q$ as $\ell(z)=s \tau_{3}=\eta / q^{5}$, and thus we can more generally write

$$
\frac{d N(z)}{d z}=\frac{N(z)}{\ell(z)} \ln 2=\frac{\ln 2}{\eta}\left(\frac{q_{0}}{N(z)}\right)^{5} N(z)
$$

since the initial phonon energy becomes spread among $N$ phonons, with average phonon momentum $q_{0} / N$. The solution of Eq. (62) is

\footnotetext{
${ }^{6}$ The present calculation is roughly consistent with the measurement of Wyatt et al. [52] in which they applied a collimated heat pulse at temperature $T_{p}$ (not the ambient helium temperature) and measured the opening angle of the phonon cone produced, finding angles of order $8^{\circ}$ for a thermal distribution of initial phonons at $T_{p}=2.2 \mathrm{~K}$.
} 


$$
N(z)=\left(1+(5 \ln 2) \frac{z}{\ell_{0}}\right)^{1 / 5}
$$

where $\ell_{0}=\eta / q_{0}^{5}$ is the mean free path of the initial phonon. For $q_{0}=0.5 \AA^{-1}$ (or phonon energy $9 \mathrm{~K}$ ), we have $\ell_{0} \sim 53 \AA$, and in a cascade of length $30 \mathrm{~cm}$, the final number of phonons in the cascade is $N \sim 42$. The mean phonon energy is $\sim 0.21 \mathrm{~K}$, an order of magnitude larger than the expected ambient temperature of $\sim 10 \mathrm{mK}$. The phonon mean free path exceeds the radius, $R$, of the helium container, only for $q \lesssim 0.014 \AA^{-1}(30 \mathrm{~cm} / R)^{1 / 5}$.

We next estimate the widening of the cascade with subsequent decays, again with the simplifying assumption that phonons split only into pairs of equal energy. We keep only the $\zeta_{A}$ term in the dispersion. Then, after $n$ splittings, the angle $\delta_{n}$ of an $n$ th-generation phonon with respect to its progenitor phonon of momentum $\vec{q}_{n-1}$ is

$$
\delta_{n}^{2} \simeq \frac{3}{2} \zeta_{A}\left(\frac{q}{2^{n-1}}\right)^{2},
$$

and its momentum is given by

$$
\vec{q}_{n}=\frac{1}{2}\left(\vec{q}_{n-1}+\delta_{n} q_{n-1} \hat{m}_{n}\right)
$$

where $\hat{m}_{n}$ is a unit vector orthogonal to $\vec{q}_{n-1}$. The opening angle measured with respect to the initial phonon $\vec{q}_{0}$ is then given by $\cos \theta_{n} \equiv \hat{q}_{n} \cdot \hat{q}_{0}$, so that to leading order,

$$
\theta_{n}^{2}=\delta_{n}^{2}+\theta_{n-1}^{2}-2 \delta_{n} \hat{q}_{0} \cdot \hat{m}_{n}
$$

When averaged over $\hat{m}_{n}$, the last term goes away, and using Eq. (64) at $q_{1}=q_{0} / 2$, we find

$$
\theta_{n}^{2}=\frac{3}{2} \zeta_{A}\left(\frac{q_{0}}{2^{n-1}}\right)^{2}+\theta_{n-1}^{2},
$$

a recursive relation with the solution

$$
\theta_{n}^{2}=2 \zeta_{A} q_{0}^{2}\left(1-\frac{1}{4^{n}}\right)
$$

This simple estimate implies that the opening angle of the cone increases with subsequent phonon decays only by a factor $\lesssim 2 / \sqrt{3}$; the basic physics is that the smaller the momentum of the phonons, the smaller the opening angle in the decay.

As is shown in Fig. 10, which assumes that the daughter phonons have equal energy, the phonon mean free path in the anomalous dispersion region, Eq. (41), increases rapidly as $2^{5 n}$, where $n$ is the generation index as the phonons split into pairs; at the same time, the angle between the daughter phonons decreases as $2^{n}$. As a result, the number of phonons in the shower depends strongly on

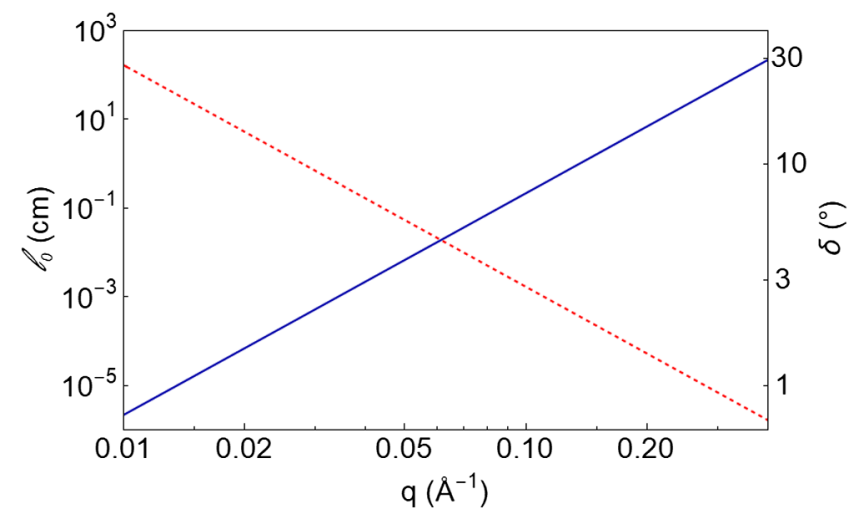

FIG. 10. The mean free path, $\ell$ (dashed, left axis), and the rms angle in degrees of the daughter phonons, $\delta$ (solid, right axis), as a function of phonon momentum, $q$ for the case of phonons split evenly in energy. Note that the mean free path varies rapidly, from a few hundred $\AA$ at $q=0.4 \AA^{-1}$ (somewhat below the maximum momentum that can decay into two phonons) to $\sim 2 \mathrm{~cm}$ at $q=0.4 / 2^{4}=0.025 \AA^{-1}$.

the initial phonon momentum and increases only slowly after traveling distances of order $1 \mathrm{~cm}$ in the detector, as shown in Fig. 11.

A detector that measures simply the total energy of an excitation at the container wall does not provide information on where the excitation was produced. The geometry of a phonon cascade, however, carries with it such information. The characteristic signature of a phonon cascade is that its energy deposition in detectors on the surface of the helium will be elliptically shaped. This signature provides discrimination against background events in the detectors. The orientation angle of the major axis of the ellipse, together with the ratio of the minor to

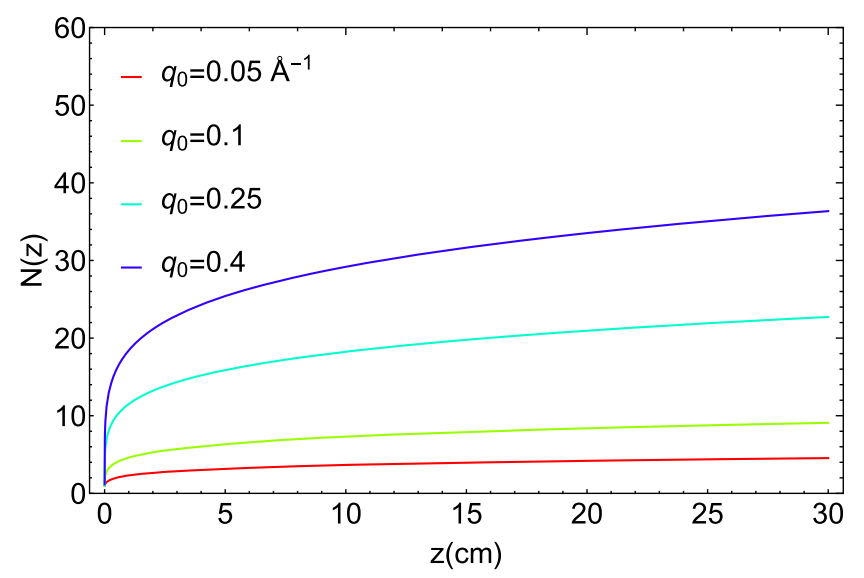

FIG. 11. The number of phonons, $N(z)$, produced as a function of path length, $z$, for a number of initial momenta, $q_{0}$, and for the case of phonons split evenly in energy. The number of phonons in the shower increases rapidly for the first few $\mathrm{cm}$ of the path and thereafter increases relatively slowly. 


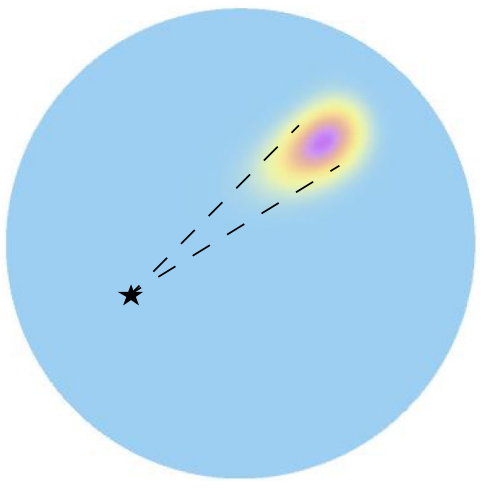

FIG. 12. A schematic illustration of the pattern of elliptical energy deposition on the surface of a spherical container of helium resulting from a phonon cascade. The dashed lines symbolically indicate the phonon cone, which expands according to Eq. (68), and the vertex of the interaction of the dark matter with the helium.

major axes, tells one the direction cosines of the original velocity vector with respect to the surface. The size of the minor axis tells one the opening angle of the phonon cascade, from which one can deduce the distance from the center of the ellipse to the original vertex; see Eq. (68). A typical pattern of energy deposition on the surface of a sphere is shown in Fig. 12. The total energy deposition is a measure of the energy transferred by the dark matter particle. With sufficient detector sensitivity on the surface, one can take advantage of the anomalous dispersion to pin down the event.

The energy of the initial phonon equals the total energy of phonons arriving at the detector, and therefore a measurement of the latter would determine the energy as well as the magnitude of the momentum of the initial phonon. A natural question to ask is whether, for an initial phonon produced with momentum in the anomalous region of the dispersion curve, the spatial distribution of secondary phonons arriving at the detector can provide information about the origin of the initial phonon. Each such event produces a pair of daughter phonons with a relatively wide opening angle and a random azimuthal orientation; subsequent splittings of the phonons in the cascade proceed with successively smaller opening angles. The overall elliptic pattern that an event would produce at the surface (schematically shown in Fig. 12 for an event with many phonons) contains information about the location of the original event, but it is a quantitative question as to whether the uncertainties obscure the significance of such location information. In principle, one would like to do a simulation, starting with a candidate dark matter mass, to determine the extent to which the event patterns at the detector surface can constrain the extracted mass, a task we leave for future work.

\section{CONCLUSIONS}

We have discussed here the processes that will occur when a low-mass dark matter particle scatters from liquid ${ }^{4} \mathrm{He}$. As we have shown, the total strength of the excitation function for states with two or more phonons is tightly constrained by sum-rule arguments; these become increasingly severe as the momentum transfer to the helium declines, and thus at lower dark matter particle masses. For dark matter masses $\lesssim 1.2 \mathrm{MeV}$, the most probable outcome is the creation of a single phonon, and this process becomes overwhelmingly dominant at $\mathrm{keV}$ masses. Moreover, at liquid pressures less than $\sim 18$ bar, this situation is made more interesting by the fact that a single phonon can decay into a cascade of lower-energy phonons. Consequently, the distinction between single-phonon and multiphonon scattering is blurred; a two-phonon state, for example, can be reached from the ground state of ${ }^{4} \mathrm{He}$ either by direct creation of two phonons or by creation of a single phonon which subsequently decays, and the net rate of production of phonon pairs is a coherent superposition of these two processes. As these arguments indicate, and as discussed in Refs. [28,29], two-phonon processes that can produce phonons in the several-meV range grow in relevance for dark matter masses $\gtrsim \mathrm{MeV}$. Our discussion complements that of Zurek and collaborators by exploring the processes relevant at lower-momentum transfers and nearer to the dispersion curve.

Our findings present both experimental challenges and opportunities. Although a thorough consideration of detection techniques is beyond the scope of the paper, we address briefly two key aspects relevant to sub-MeV dark matter. In this mass regime, the bulk of the events will appear in the form of creation of single phonons, pointing to the need to detect phonons of energy $1 \mathrm{meV}$ or lower. Above $\omega\left(q_{c}\right) \sim 0.7 \mathrm{meV}$ such phonons travel ballistically, and can be detected via surface evaporation or direct absorption by bolometric detectors [32]. This technique could be extended to lower energies by using a pressurized helium vessel to suppress anomalous dispersion.

At low pressures, phonons of energy below $\sim 0.7 \mathrm{meV}$ will decay into a cascade of even lower-energy phonons, so it becomes necessary to develop methods to detect such showers. Such decays will occur even if a pair of such phonons is created initially. If detectable, the resulting shower would contain information about the direction and magnitude of the total momentum imparted to the helium, in much the same way as the direction of the incoming primary that creates a cosmic ray shower is determined. That said, even with sensitive detectors, such information would be very difficult to obtain. The probability of a phonon escaping from liquid $\mathrm{He}$ and depositing its energy in a detector is substantially reduced by Kapitza resistance: Tanatarov et al. [70] note $\sim 1.5 \%$ transmission at best, and then only at normal incidence. 
To assess detection possibilities, it is instructive to consider detection of the energy deposited by low-energy photons. In an example from this rapidly developing field, the authors of Ref. [71] used a quantum capacitance detector to measure, with high efficiency, single photons from a $5 \mathrm{~K}$ blackbody source which has a most probable photon energy of about $2 \mathrm{meV}$. Similar techniques may point the way to direct detection of low-energy phonons. Although current progress is encouraging, broadly speaking, the use of superfluid ${ }^{4} \mathrm{He}$ detectors for dark matter detection awaits further development of detection techniques. With improved detection capabilities, superfluid ${ }^{4} \mathrm{He}$ may have the potential not only to record events produced by low-mass dark matter particles, but also to pin down the particle mass and interaction cross section with baryonic matter.

\section{ACKNOWLEDGMENTS}

This research was supported in part by NSF Grants No. PHY1714042 and No. PHY18-22502. The work of J.S. is supported by DOE CAREER Grant No. DESC0017840. Author G. B. is grateful to the Aspen Center for Physics, supported by NSF Grant No. PHY1607611, and to the Niels Bohr International Academy, where part of this research was done.

\section{APPENDIX A: RELATION OF THE DYNAMICAL STRUCTURE FUNCTION TO THE ${ }^{4}$ He DENSITY CORRELATION FUNCTION}

We recall here the relation of $S(q, \omega)$ for real frequency $\omega$ to the density-density correlation function in the complex frequency plane. In general, at temperature $T$, the density correlations $\langle\rho \rho\rangle(q, z)$, where in standard condensed matter notation $z$ is the complex frequency, are defined by [72]

$$
\begin{aligned}
& \left\langle\rho(\vec{r}, t) \rho\left(\vec{r}^{\prime}, t^{\prime}\right)\right\rangle \\
& \quad=i T \sum_{n=-\infty}^{\infty} e^{\left.-i z_{n}\left(t-t^{\prime}\right)\right)} \int \frac{d^{3} q}{(2 \pi)^{3}} e^{i \vec{q} \cdot\left(\vec{r}-\vec{r}^{\prime}\right)}\langle\rho \rho\rangle\left(q, z_{n}\right),
\end{aligned}
$$

where $z_{n}=2 \pi i T n$ are the Matsubara frequencies. Then $\langle\rho \rho\rangle(q, z)$, the analytic continuation of $\langle\rho \rho\rangle\left(q, z_{n}\right)$ to the complex frequency $z$ plane, has the form

$$
\langle\rho \rho\rangle(q, z)=\frac{n_{4} q^{2} / m_{4}}{z^{2}-\Pi(q, z)},
$$

and is given in terms of the structure function by

$$
\begin{aligned}
\langle\rho \rho\rangle(q, z) & =n_{4} \int_{-\infty}^{\infty} d \omega \frac{S(q, \omega)-S(q,-\omega)}{z-\omega} \\
& =2 n_{4} \int_{-\infty}^{\infty} d \omega \frac{\omega S(q, \omega)}{z^{2}-\omega^{2}} .
\end{aligned}
$$

Comparison of the large- $z$ limit of Eqs. (A4) and (A2) yields the $f$-sum rule, Eq. (10). At zero temperature, $S$ vanishes for negative frequency. The following appendix discusses $S(q, \omega)$ at finite temperature.

We write $\Pi$ in terms of its real and imaginary parts, $\Pi(q, \omega+i \epsilon)=\Re \Pi+i \Im \Pi$, where $+i \epsilon$, with $\epsilon \rightarrow 0$, indicates a limit to the real axis from the upper half-plane. In this limit, $\Im \Pi \leq 0$, for $\omega \geq 0$. The imaginary parts of Eqs. (A3) and (A2) then imply

$$
S(q, \omega)=\frac{q^{2}}{\pi m_{4}} \frac{-\Im \Pi}{\left(\omega^{2}-\Re \Pi\right)^{2}+\Im \Pi^{2}} .
$$

At low $q$, where single-phonon excitations dominate, $\Pi$ has the structure

$$
\Pi(q, \omega)=\omega(q)^{2}+\mathcal{R}(q, \omega)-i \omega(q) \gamma(q, \omega)
$$

where $\mathcal{R}$ is real; then $S(q, \omega)$ for $\omega \geq 0$ becomes

$S(q, \omega)=\frac{q^{2}}{\pi m_{4}} \frac{\omega(q) \gamma(q, \omega)}{\left(\omega^{2}-\omega(q)^{2}-\mathcal{R}\right)^{2}+\omega(q)^{2} \gamma(q, \omega)^{2}}$.

The function $\gamma(q, \omega)$, which is non-negative, determines the damping rate of the density excitations. With $\mathcal{R}$ in the denominator of Eq. (A7) neglected, and $\gamma \rightarrow \gamma_{2}$ in the numerator, Eq. (A7), derived from the structure of the density-density correlation function, reduces to the "one-to-two" phonon contribution to Eq. (45) with $\gamma_{2}$ given by Eq. (33).

In the limit $\gamma \rightarrow 0$, the structure function for $\omega \geq 0$ reduces to

$$
\begin{aligned}
S(q, \omega) & \left.=\frac{q^{2}}{m_{4}} \delta\left(\omega^{2}-\omega(q)^{2}-\mathcal{R}(q, \omega)\right)^{2}\right) \\
& =\frac{q^{2}}{2 m_{4} \omega(q)_{R}} \frac{\delta\left(\omega-\omega(q)_{R}\right)}{\left(1-\partial \mathcal{R} /\left.\partial \omega^{2}\right|_{\omega=\omega(q)_{R}}\right)} .
\end{aligned}
$$

The term $\mathcal{R}(q, \omega)$ serves to renormalize the excitation energy from $\omega(q)$ to the solution, $\omega(q)_{R}$, of $\omega^{2}=$ $\omega(q)^{2}+\mathcal{R}(q, \omega)$. We neglect such renormalization effects throughout. The factor $Z(q)$ in the decomposition [Eq. (11)] is the coefficient of the $\delta$ function in Eq. (A8). Renormalization of the excitation energy and the decrease of the contribution of the single-phonon peak to the $f$-sum rule are intimately related. In the limit $\gamma \rightarrow 0$ with $\mathcal{R}$ neglected, the structure function reduces to the single-phonon result, Eq. (39). 


\section{APPENDIX B: $S(q, \omega)$ IN HeII AT FINITE TEMPERATURE}

Since in general at finite $T, S_{T}(q,-\omega)=e^{-\omega / T} S_{T}(q, \omega)$, we can write

$$
S_{T}(q, \omega)=(1+n(\omega)) B(q, \omega),
$$

where $n(\omega)=1 /\left(e^{\omega / T}-1\right)$ is the Bose occupation factor at energy $\omega$, and $B(q, \omega)$ is odd in $\omega$ and non-negative for $\omega>0$.

The $f$-sum rule becomes

$$
\begin{gathered}
\int_{-\infty}^{\infty} d \omega \omega(1+n(\omega)) B(q, \omega) \\
=\int_{0}^{\infty} d \omega \omega B(q, \omega)=\frac{q^{2}}{2 m_{4}},
\end{gathered}
$$

while the static structure function becomes

$$
\begin{aligned}
S_{T}(q) & =\int_{-\infty}^{\infty} d \omega(1+n(\omega)) B(q, \omega) \\
& =\int_{0}^{\infty} d \omega(1+2 n(\omega)) B(q, \omega) .
\end{aligned}
$$

Separating out the single-phonon contribution to $B$, we write for $\omega>0$,

$$
B(q, \omega)=Z(q) \delta(\omega-\omega(q))+B_{M}(q, \omega),
$$

where $B_{M} \geq 0$ is the multiexcitation contribution. Then

$$
\begin{aligned}
S_{T}(q, \omega)= & (1+n(\omega(q))) Z(q) \delta(\omega-\omega(q)) \\
& +(1+n(\omega)) B_{M}(q, \omega),
\end{aligned}
$$

and the weight of the phonon pole at finite temperature is

$$
Z_{T}(q)=(1+n(\omega(q)) Z(q) .
$$

The $f$-sum rule then implies that the multiexcitation contribution obeys

$$
\int_{0}^{\infty} d \omega \omega B_{M}(q, \omega)=\frac{q^{2}}{2 m_{4}}-\omega(q) Z(q) .
$$

In addition,

$$
\begin{aligned}
S_{T}(q)= & (1+2 n(\omega(q))) Z(q) \\
& +\int_{0}^{\infty} d \omega(1+2 n(\omega)) B_{M}(q, \omega) .
\end{aligned}
$$

This equation enables us to place bounds on $S_{T}(q)$. Since $(1+2 n(\omega))$ is a decreasing function of $\omega$, and the support of $B_{M}(q, \omega)$ is essentially for $\omega \geq \omega(q)$, we have, with Eq. (B7),

$$
\begin{aligned}
& \int_{0}^{\infty} d \omega(1+2 n(\omega)) B_{M}(q, \omega) \\
& \quad<(1+2 n(\omega(q))) \int_{0}^{\infty} d \omega B_{M}(q, \omega) \frac{\omega}{\omega(q)} \\
& \quad=(1+2 n(\omega(q)))\left(\frac{q^{2}}{2 m_{4} \omega(q)}-Z(q)\right) .
\end{aligned}
$$

Thus, from Eq. (B8) we have

$$
S_{T}(q) \leq \frac{q^{2}}{2 m_{4} \omega(q)}(1+2 n(\omega(q)))
$$

while if we neglect the multiexcitation contribution to $S(q)$, we see that

$$
S_{T}(q) \geq Z(q)[1+2 n(\omega(q)] .
$$

Altogether,

$$
Z(q) \leq \frac{S_{T}(q)}{1+2 n(\omega(q)} \leq \frac{q^{2}}{2 m_{4} \omega(q)}
$$

Liu and Woo [60] give the expansions at $T=0$ :

$$
S(q)=\frac{x}{2}\left(1-1.63 x^{2}+1.42 x^{3}+0.51 x^{4}\right),
$$

$Z(q)=\frac{x}{2}\left(1-1.63 x^{2}-0.78 x^{3}+0.51 x^{4}-2.46 x^{5}\right)$,

where $x=q / m_{4} s$.

For small $q$, Eq. (B12) becomes

$1-z_{2}\left(\frac{q}{m_{4} s}\right)^{2} \leq \frac{S(q)}{(1+2 n(\omega(q)))\left(q / 2 m_{4} s\right)} \leq 2$,

with $z_{2} \simeq 1.63$. Then, to order $x^{4}$,

$$
S(q)-Z(q)=1.1\left(\frac{q}{m_{4} s}\right)^{3},
$$

which, to within the error bars, is consistent with the $Z(q)$ data from Cowley and Woods, lying below that for $S(q)$ given by Robkoff and Hallock, as seen in Fig. 4.

We note furthermore that the single-phonon contribution to $S_{T}(q)$ at finite temperature is $(1+2 n(\omega(q))) Z(q)$, while the weight of the pole is $Z_{T}(q)=(1+n(\omega(q))) Z(q)$, where $Z(q)$ is essentially the zero-temperature weight of the pole. The finite-temperature effects on $S_{T}(q)$ are twice as large as those on $Z_{T}(q)$. 


\section{APPENDIX C: MATRIX ELEMENT FOR PRODUCTION OF TWO PHONONS}

We review how the matrix element for the two-phonon process in Fig. 3(c) is constrained, for small momentum transfers, by the particle number conservation law [73]. With the basic interaction between a dark matter particle and the helium atoms represented by a contact interaction, Eq. (5), the ${ }^{4} \mathrm{He}$ part of the matrix element is proportional to the Fourier transform $\rho_{-\vec{q}}$ of the density operator. Particle number conservation, $\partial \rho_{-\vec{q}} / \partial t-i \vec{q} \cdot \vec{j}_{-\vec{q}}=0$, where $\rho_{-\vec{q}}$ and $\vec{j}_{-\vec{q}}$ are the Fourier transforms of the number and number current density operators, implies that the matrix element of $\rho$ between the initial and final states obeys

$$
\left\langle f\left|\rho_{-\vec{q}}\right| 0\right\rangle=\frac{\vec{q} \cdot\left\langle f\left|\vec{j}_{-\vec{q}}\right| 0\right\rangle}{E_{f}-E_{0}} .
$$

The E's are the energies of the initial and final states. Since the system is translationally invariant, the energy eigenstates may be also be taken to be eigenstates of the total momentum, which implies that, for $\vec{q} \rightarrow 0, \vec{j}_{-\vec{q}}$ has no offdiagonal matrix elements. Thus, one expects that for small $q$, and for different initial- and final-state energies, $\left\langle f\left|\vec{j}_{-\vec{q}}\right| 0\right\rangle \propto q^{\alpha} \hat{q}$, with $\alpha>0$ and

$$
\left\langle f\left|\rho_{-\vec{q}}\right| 0\right\rangle \sim \frac{q^{1+\alpha}}{E_{f}-E_{0}},
$$

when the magnitudes of the phonon momenta in the final state are held fixed. As $q \rightarrow 0$, the energy denominator remains finite for two (or more) phonon excitations. Analyticity in $q$ implies that the smallest value of $\alpha$ is unity, and so $\left\langle f\left|\rho_{-\vec{q}}\right| 0\right\rangle \propto q^{2}$, as in Eq. (51). By contrast, the matrix element of the current operator for the creation of a single phonon is anomalous, varying as $q^{1 / 2}$, as explained in Ref. [73].

We now estimate the dimensionless matrix element $\mathcal{M}$ using the sum-rule result, Eq. (23). We let $q \rightarrow 0$ in the integral in Eq. (54), so that $\vec{q}_{2}=-\vec{q}_{1}$, i.e., the phonons are back to back; then

$$
\begin{aligned}
S_{2}(q & \rightarrow 0, \omega) \\
= & \frac{3 q^{4}}{8 \pi k_{D}^{3} m_{4}^{2}} \int d^{3} q_{1} \delta\left(\omega-2 \omega\left(q_{1}\right)\right)\left|\left\langle\vec{q}_{1},-\vec{q}_{1}|M| 0\right\rangle\right|^{2} \\
= & \frac{3 q^{4}}{16 k_{D}^{3} m_{4}^{2} s^{3}}\left(\frac{1}{2}(u-1)+\frac{\mathcal{M} \omega}{s k_{D}}\right)^{2},
\end{aligned}
$$

with the factor $(u-1)$ for back-to-back phonons.

Including phonons up to momenta $s k_{D}$, we find the contribution to the $f$-sum rule from $S_{2}(q, \omega)$ for small $q$ :

$$
\begin{aligned}
& \int_{0}^{2 s k_{D}} d \omega \omega S_{2}(q, \omega) \\
& \quad=\frac{3 q^{4}}{4 k_{D} m_{4}^{2} s}\left(\frac{1}{8}(u-1)^{2}+\frac{2}{3}(u-1) \mathcal{M}+\mathcal{M}^{2}\right) .
\end{aligned}
$$

Comparing with Eq. (13), we have

$$
\frac{1}{8}(u-1)^{2}+\frac{2}{3}(u-1) \mathcal{M}+\mathcal{M}^{2}=\frac{2}{3} z_{2} \frac{k_{D}}{m_{4} s} ;
$$

numerically, $\mathcal{M}=-1.47$ or +0.25 . Using Eq. (C5), we find that the combination of terms, $\mathcal{M}^{2}+(3 \pi / 16)$ $\mathcal{M}(u-1)+(u-1)^{2} / 10$ in Eq. (57) is 0.7 or 0.9 .
[1] G. Bertone and D. Hooper, Rev. Mod. Phys. 90, 045002 (2018).

[2] M. W. Goodman and E. Witten, Phys. Rev. D 31, 3059 (1985).

[3] G. Jungman, M. Kamionkowski, and K. Griest, Phys. Rep. 267, 195 (1996).

[4] M. Schumann, J. Phys. G 46, 103003 (2019).

[5] J. Alexander et al., arXiv:1608.08632.

[6] M. Battaglieri et al., arXiv:1707.04591.

[7] M. Viel, G. D. Becker, J. S. Bolton, and M. G. Haehnelt, Phys. Rev. D 88, 043502 (2013).

[8] R. Essig, J. Mardon, and T. Volansky, Phys. Rev. D 85, 076007 (2012).

[9] P.W. Graham, D.E. Kaplan, S. Rajendran, and M. T. Walters, Phys. Dark Universe 1, 32 (2012).
[10] R. Essig, M. Fernandez-Serra, J. Mardon, A. Soto, T. Volansky, and T. T. Yu, J. High Energy Phys. 05 (2016) 046.

[11] R. Agnese et al. (SuperCDMS Collaboration), Phys. Rev. Lett. 121, 051301 (2018); 122, 069901(E) (2019).

[12] O. Abramoff et al. (SENSEI Collaboration), Phys. Rev. Lett. 122, 161801 (2019).

[13] A. Aguilar-Arevalo et al. (DAMIC Collaboration), Phys. Rev. Lett. 123, 181802 (2019).

[14] R. Essig, T. Volansky, and T. T. Yu, Phys. Rev. D 96, 043017 (2017).

[15] P. Agnes et al. (DarkSide Collaboration), Phys. Rev. Lett. 121, 111303 (2018).

[16] E. Aprile et al. (XENON Collaboration), Phys. Rev. Lett. 123, 251801 (2019). 
[17] S. Derenzo, R. Essig, A. Massari, A. Soto, and T. T. Yu, Phys. Rev. D 96, 016026 (2017).

[18] C. Blanco, J. I. Collar, Y. Kahn, and B. Lillard, Phys. Rev. D 101, 056001 (2020).

[19] R. Essig, J. Pérez-Rios, H. Ramani, and O. Slone, Phys. Rev. Research 1, 033105 (2019).

[20] R. Budnik, O. Chesnovsky, O. Slone, and T. Volansky, Phys. Lett. B 782, 242 (2018).

[21] D. Baxter, Y. Kahn, and G. Krnjaic, Phys. Rev. D 101, 076014 (2020).

[22] R. Essig, J. Pradler, M. Sholapurkar, and T. T. Yu, Phys. Rev. Lett. 124, 021801 (2020).

[23] Y. Hochberg, Y. Zhao, and K. M. Zurek, Phys. Rev. Lett. 116, 011301 (2016); Y. Hochberg, M. Pyle, Y. Zhao, and K. M. Zurek, J. High Energy Phys. 08 (2016) 057; Y. Hochberg, I. Charaev, S. W. Nam, V. Verma, M. Colangelo, and K. K. Berggren, Phys. Rev. Lett. 123, 151802 (2019).

[24] Y. Hochberg, Y. Kahn, M. Lisanti, K. M. Zurek, A. G. Grushin, R. Ilan, S. M. Griffin, Z.-F. Liu, S. F. Weber, and J. B. Neaton, Phys. Rev. D 97, 015004 (2018).

[25] S. Knapen, T. Lin, M. Pyle, and K. M. Zurek, Phys. Lett. B 785, 386 (2018).

[26] S. M. Griffin, K. Inzani, T. Trickle, Z. Zhang, and K. M. Zurek, Phys. Rev. D 101, 055004 (2020).

[27] W. Guo and D. N. McKinsey, Phys. Rev. D 87, 115001 (2013).

[28] K. Schutz and K. M. Zurek, Phys. Rev. Lett. 117, 121302 (2016).

[29] S. Knapen, T. Lin, and K. M. Zurek, Phys. Rev. D 95, 056019 (2017).

[30] H. J. Maris, G. M. Seidel, and D. Stein, Phys. Rev. Lett. 119, 181303 (2017).

[31] T. M. Ito and G. M. Seidel, Phys. Rev. C 88, 025805 (2013).

[32] S. A. Hertel, A. Biekert, J. Lin, V. Velan, and D. N. McKinsey, Phys. Rev. D 100, 092007 (2019).

[33] C. E. Campbell, E. Krotscheck, and T. Lichtenegger, Phys. Rev. B 91, 184510 (2015).

[34] S. T. Beliaev, Zh. Eksp. Teor. Fiz. 34, 433 (1958) [Sov. Phys. JETP 7, 299 (1958)].

[35] H. J. Maris, Rev. Mod. Phys. 49, 341 (1977).

[36] F. Acanfora, A. Esposito, and A. D. Polosa, Eur. Phys. J. C 79, 549 (2019).

[37] A. Caputo, A. Esposito, and A. D. Polosa, J. Phys. Conf. Ser. 1468, 012060 (2020).

[38] A. Caputo, A. Esposito, and A. D. Polosa, Phys. Rev. D 100, 116007 (2019).

[39] J. I. Read, J. Phys. G 41, 063101 (2014).

[40] M. Benito, A. Cuoco, and F. Iocco, J. Cosmol. Astropart. Phys. 03 (2019) 033.

[41] P. F. de Salas, K. Malhan, K. Freese, K. Hattori, and M. Valluri, J. Cosmol. Astropart. Phys. 10 (2019) 037.

[42] J. D. Lewin and P. F. Smith, Astropart. Phys. 6, 87 (1996).

[43] C. Kelso, C. Savage, M. Valluri, K. Freese, G. S. Stinson, and J. Bailin, J. Cosmol. Astropart. Phys. 08 (2016) 071.

[44] M. C. Smith et al., Mon. Not. R. Astron. Soc. 379, 755 (2007).
[45] J. Bovy et al., Astrophys. J. 759, 131 (2012).

[46] K. Freese, M. Lisanti, and C. Savage, Rev. Mod. Phys. 85, 1561 (2013).

[47] M. Kuhlen, N. Weiner, J. Diemand, P. Madau, B. Moore, D. Potter, J. Stadel, and M. Zemp, J. Cosmol. Astropart. Phys. 02 (2010) 030.

[48] M. Lisanti and D. N. Spergel, Phys. Dark Universe 1, 155 (2012).

[49] C. A. J. O'Hare, C. McCabe, N. W. Evans, G. Myeong, and V. Belokurov, Phys. Rev. D 98, 103006 (2018).

[50] R. J. Donnelly, J. A. Donnelly, and R. N. Hills, J. Low Temp. Phys. 44, 471 (1981).

[51] D. S. Greywall, Phys. Rev. B 23, 2152 (1981).

[52] R. A. Sherlock, N. G. Mills, and A. F. G. Wyatt, J. Phys. C 8, 2575 (1975).

[53] R. Sridhar, Phys. Rep. 146, 259 (1987).

[54] M. P. Kemoklidze and L. P. Pitaevskii, Zh. Eksp. Teor. Fiz. 59, 2187 (1970) [Sov. Phys. JETP 32, 1183 (1971)].

[55] K. Beauvois, H. Godfrin, E. Krotscheck, and R. E. Zillich, J. Low Temp. Phys. 197, 113 (2019).

[56] H. R. Glyde, Rep. Prog. Phys. 81, 014501 (2018).

[57] W. G. Stirling, in The 75th Jubilee Conf. on Helium-4, edited by J. G. M. Armitage (World Scientific, Singapore, 1983), p. 109.

[58] L. D. Landau and I. M. Khalatnikov, Zh. Eksp. Teor. Fiz. 19, 637 (1949) [English translation in The Collected Papers of L. D. Landau, edited by (Gordon and Breach, New York, 1965), p. 494.

[59] D. Pines and C.-W. Woo, Phys. Rev. Lett. 24, 1044 (1970).

[60] Y.-R. Lin Liu and C.-W. Woo, J. Low Temp. Phys. 14, 317 (1974).

[61] H. N. Robkoff and R. B. Hallock, Phys. Rev. B 24, 159 (1981).

[62] R. A. Cowley and A. D. B. Woods, Can. J. Phys. 49, 177 (1971).

[63] E. C. Svensson, V. F. Sears, A. D. B. Woods, and P. Martel, Phys. Rev. B 21, 3638 (1980).

[64] G. Baym and C. Ebner, Phys. Rev. 164, 235 (1967), Eq. (22).

[65] P. C. Hohenberg and P. C. Martin, Ann. Phys. (N.Y.) 34, 291 (1965).

[66] E. M. Lifshitz and L. P. Pitaevskii, Physical Kinetics (Pergamon, Oxford, 1981), p. 328.

[67] G. Baym, D. H. Beck, and C. J. Pethick, J. Low Temp. Phys. 178, 200 (2015).

[68] D. Benin, Phys. Rev. B 11, 145 (1975).

[69] See, e.g., Ref. [66], p. 38.

[70] I. V. Tanatarov, I. N. Adamenko, K. E. Nemchenko, and A. F. G. Wyatt, J. Low Temp. Phys. 159, 549 (2010).

[71] P. M. Echternach, B. J. Pepper, T. Reck, and C. M. Bradford, Nat. Astron. 2, 90 (2018).

[72] L. P. Kadanoff and G. Baym, Quantum Statistical Mechanics (W. A. Benjamin, New York, 1962).

[73] P. Nozières and D. Pines, The Theory of Quantum Liquids (Addison-Wesley, Reading, Massachusetts, 1990), Vol. II, Sec. II. 4. 